\title{
LITTLEWOOD-PALEY SPACES
}

\author{
KWOK-PUN HO
}

\begin{abstract}
We introduce the Littlewood-Paley spaces in which the Lebesgue spaces, the Hardy spaces, the Orlicz spaces, the Lorentz-Karamata spaces, the r.-i. quasi-Banach function spaces and the Morrey spaces reside. The Littlewood-Paley spaces provide a unified framework for the study of some important function spaces arising in analysis.
\end{abstract}

\section{Introduction and Preliminaries}

In this paper, we further develop the Littlewood-Paley characterization of function spaces by introducing a new class of function spaces, namely, the Littlewood-Paley spaces (see Definition 2.1). This extends a line of research which includes the Triebel-Lizorkin spaces [23], [24], [56], the LorentzKaramata spaces [16], [44], [45], the Orlicz spaces [49] and their generalizations, the rearrangement-invariant (r.-i.) quasi-Banach function spaces [5], [42], [46], the variable Lebesgue spaces [14], [15] and the Morrey type spaces [12], [32], [39], [52], [53].

The general theory for the Littlewood-Paley spaces is given in Sections 2 and 3. The applications of the general theory to some well-known function spaces are presented in Sections 4 and 5. We find that the notions of the UMD property and the $p$-convexification are involved in the study of the LittlewoodPaley spaces.

Let $\mathscr{M}\left(\mathrm{R}^{n}\right)$ be the set of Lebesgue measurable functions on $\mathrm{R}^{n}$. Let $\mathcal{M}_{0}\left(\mathrm{R}^{n}\right)$ be the class of functions in $\mathscr{M}\left(\mathrm{R}^{n}\right)$ that are finite almost everywhere. Let $\mathscr{S}\left(\mathrm{R}^{n}\right)$ and $\mathscr{S}^{\prime}\left(\mathrm{R}^{n}\right)$ be the classes of tempered functions and Schwartz distributions, respectively. Let $\mathscr{S}_{0}\left(\mathrm{R}^{n}\right)=\left\{\varphi \in \mathscr{S}\left(\mathrm{R}^{n}\right): \int x^{\gamma} \varphi(x) d x=0, \gamma \in \mathrm{N}^{n}\right\}$. Let $\mathscr{P}$ be the class of polynomials on $\mathrm{R}^{n}$ and $\mathscr{C}$ be the class of constant functions.

Definition 1.1. Let $\mu$ be the Lebesgue measure on $\mathrm{R}^{n}$ or the counting measure on Z. Let $\mathscr{M}(\mu)$ be the set of $\mu$-measurable functions. A mapping $\|\cdot\|: \mathscr{M}(\mu) \rightarrow[0, \infty]$ is called a regular quasi-norm if for all $f, g \in \mathscr{M}(\mu)$ and $\left\{f_{n}\right\}_{n \in \mathbb{N}} \subset \mathcal{M}(\mu),\|\cdot\|$ satisfies:

(1) $\|\cdot\|$ is a complete quasi-norm;

Reveived 27 April 2009, in final form 5 November 2009. 
(2) $|g| \leq|f|, \mu$-almost everywhere implies $\|g\| \leq\|f\|$ and $\|f\|=0$ iff $f=0$ a.e.;

(3) if $E$ is a $\mu$-measurable bounded set, then $\left\|\chi_{E}\right\|<\infty$; and

(4) $0 \leq f_{n} \uparrow f \mu$-a.e. and $\sup _{n}\left\|f_{n}\right\|<\infty$ imply $\left\|f_{n}\right\| \uparrow\|f\|$.

Definition 1.2. We call a quasi-Banach space $\mathscr{B}_{f} \subseteq \mathcal{M}\left(\mathrm{R}^{n}\right)$ a regular function space if $\|\cdot\|_{\mathscr{B}_{f}}$ is a regular quasi-norm, $\mathscr{B}_{f}=\left\{f \in \mathscr{M}\left(\mathrm{R}^{n}\right):\|f\|_{\mathscr{B}_{f}}<\right.$ $\infty\}$ and $(1+|x|)^{-\kappa} \in \mathscr{B}_{f}$ for some $\kappa>0$.

According to Definition 1.2, $\chi_{E} \in \mathscr{B}_{f}$ when $E$ is a bounded Lebesgue measurable set. Hence, condition (2) of Definition 1.1 assures that $\mathscr{B}_{f} \subset$ $\mathcal{M}_{0}\left(\mathrm{R}^{n}\right)$. We need the condition, $(1+|x|)^{-\kappa} \in \mathscr{B}_{f}$ for some $\kappa>0$, to show that $\mathscr{S}_{0}\left(\mathrm{R}^{n}\right)$ is a subset of our Littlewood-Paley spaces (see Proposition 2.2).

There are a lot of examples of regular function spaces. In fact, any Banach function space on $\mathrm{R}^{n}$ (see [5], Chapter 1, Definitions 1.1 and 1.3) satisfying the property: $\|f(\cdot)\|_{\mathscr{B}_{f}}=\|f(\cdot+a)\|_{\mathscr{B}_{f}}$, for any $a \in \mathrm{R}^{n}, f \in \mathscr{B}_{f}$, is a regular function space. In Theorem 4.8, we prove that any r.-i. quasi-Banach function space on $\mathrm{R}^{n}$ is a regular function space.

Definition 1.3. We call a quasi-Banach space $\mathscr{B}_{s} \subseteq\left\{\left\{a_{i}\right\}_{i \in Z}: a_{i} \in \mathrm{C}\right\}$ a regular sequence space if $\|\cdot\|_{\mathscr{B}_{s}}$ is a regular quasi-norm, $\mathscr{B}_{s}=\left\{\left\{a_{i}\right\}_{i \in \mathrm{Z}}\right.$ : $\left.\left\|\left\{a_{i}\right\}_{i \in Z}\right\|_{\mathscr{B}_{s}}<\infty\right\}$ and

(1) for any $l \in Z$ and $\left\{a_{i}\right\}_{i \in Z} \in \mathscr{B}_{s}$, we have $\left\{a_{i+l}\right\}_{i \in Z} \in \mathscr{B}_{s}$ with $\left\|\left\{a_{i+l}\right\}_{i \in Z}\right\|_{\mathscr{B}_{s}}$ $=\left\|\left\{a_{i}\right\}_{i \in Z}\right\|_{\mathscr{B}_{s}} ;$

(2) $\left\|\left\{f_{i}\right\}_{i \in Z}\right\|_{\mathscr{B}_{s}} \in \mathcal{M}\left(\mathrm{R}^{n}\right)$ if $\left\{f_{i}\right\}_{i \in Z} \subset \mathscr{M}\left(\mathrm{R}^{n}\right)$.

A regular quasi-norm is a lattice quasi-norm (see [46], p. 20). Thus, regular function spaces and regular sequence spaces are quasi-Banach lattices.

If $\mathscr{B}_{S}$ is a Banach sequence space (Banach function space on Z with counting measure) with separable associate space (for the definition of associate space, see [5], Chapter 1, Definition 2.3), then the Lorentz-Luxemburg theorem (see [5], Chapter 1, Theorem 2.7) asserts that $\left\|\left\{f_{i}(x)\right\}_{i \in Z}\right\|_{\mathscr{B}_{s}}=$ $\sup _{\left\{b_{i}\right\}_{i \in Z} \in \mathscr{D}} \sum_{i \in Z} f_{i}(x) b_{i},\left\{f_{i}\right\}_{i \in Z} \subset \mathcal{M}\left(\mathrm{R}^{n}\right)$ where $\mathscr{D}$ is a countable dense subset of the unit ball of the associate space. Therefore, $\mathscr{B}_{S}$ satisfies item (2) of Definition 1.3. In particular, any r.-i. reflexive Banach sequence space is a regular sequence space.

Item (1) in Definition 1.3 is used in the proofs of Proposition 2.2 and Theorem 3.1. Item (2) guarantees that the vector space $\mathscr{B}$ in the subsequent definition is well-defined.

Definition 1.4. Let $\mathscr{B}_{s}$ be a regular sequence space and $\mathscr{B}_{f}$ be a regular function space. We define the vector space $\mathscr{B}$ as

$$
\mathscr{B}=\left\{\left\{f_{i}\right\}_{i \in Z}: f_{i} \in \mathscr{M}_{0}\left(\mathrm{R}^{n}\right),\left\{f_{i}\right\}_{i \in Z} \in \mathscr{B}_{s} \text { a.e. and }\left\|\left\{f_{i}\right\}_{i \in Z}\right\|_{\mathscr{B}_{s}} \in \mathscr{B}_{f}\right\}
$$


and equip $\mathscr{B}$ with the quasi-norm $\|f\|_{\mathscr{B}}=\|\|\left\{f_{i}\right\}_{i \in Z}\left\|_{\mathscr{B}_{s}}\right\|_{\mathscr{B}_{f}}$. We endow $\mathscr{B}$ with the following partial ordering: $f \leq g$, if and only if $f_{i} \leq g_{i}$ a.e., $i \in \mathbf{Z}$, $f=\left\{f_{i}\right\}_{i \in Z}, g=\left\{g_{i}\right\}_{i \in Z} \in \mathscr{B}$.

For any sequence of locally integrable functions $f=\left\{f_{i}\right\}_{i \in Z}$, define $|f|=$ $\left\{\left|f_{i}\right|\right\}_{i \in z}$. If $\mathscr{B}_{s}$ is a regular sequence space and $\mathscr{B}_{f}$ is a regular function space, then $\|\cdot\|_{\mathscr{B}}$ is a quasi-norm satisfying

$$
|f| \leq|g| \quad \text { and } \quad g \in \mathscr{B} \Rightarrow f \in \mathscr{B} \quad \text { and } \quad\|f\|_{\mathscr{B}} \leq\|g\|_{\mathscr{B}},
$$

where $f=\left\{f_{i}\right\}_{i \in Z}$ and $g=\left\{g_{i}\right\}_{i \in Z}$.

Definition 1.5. Let $0<p<\infty$. Let $\mathscr{B}_{s}$ be a regular sequence space and $\mathscr{B}_{f}$ be a regular function space. We define the quasi-Banach spaces $\mathscr{B}_{s}^{p}$ and $\mathscr{B}_{f}^{p}$ by the $1 / p$-th powers ( $p$-convexifications) of $\mathscr{B}_{s}$ and $\mathscr{B}_{f}$, respectively. That is,

$$
\mathscr{B}_{s}^{p}=\left\{\left\{a_{i}\right\}_{i \in \mathrm{Z}}: a_{i} \in \mathrm{C},\left\{\left|a_{i}\right|^{p}\right\}_{i \in \mathrm{Z}} \in \mathscr{B}_{s}\right\}
$$

and

$$
\mathscr{B}_{f}^{p}=\left\{f \in \mathscr{M}_{0}:|f|^{p} \in \mathscr{B}_{f}\right\} ;
$$

and $\left\|\left\{a_{i}\right\}_{i \in Z}\right\|_{\mathscr{B}_{s}^{p}}=\left\|\left\{\left|a_{i}\right|^{p}\right\}_{i \in Z}\right\|_{\mathscr{B}_{s}}^{1 / p}$ and $\|f\|_{\mathscr{B}_{f}^{p}}=\left\||f|^{p}\right\|_{\mathscr{B}_{f}}^{1 / p}$.

For the basic properties of $\mathscr{B}_{s}^{p}$ and $\mathscr{B}_{f}^{p}$, the reader is referred to [46], Section 2.2. In addition, $\mathscr{B}_{s}^{p}$ and $\mathscr{B}_{f}^{p}$ are a regular sequence space and a regular function space, respectively.

LEMMA 1.6. If $\mathscr{B}_{s}$ is a regular sequence space and $\mathscr{B}_{f}$ is a regular function space, then there exists a $\rho>0$ such that for any $\left\{F_{i}\right\}_{i \in \mathrm{N}} \subset \mathscr{B}, \sum_{i \in \mathrm{N}}\left\|F_{i}\right\|_{\mathscr{B}}^{\rho}<$ $\infty \Rightarrow \sum_{i \in \mathrm{N}} F_{i} \in \mathscr{B}$.

Proof. As $\|\cdot\|_{\mathscr{B}_{s}}$ is a quasi-norm, the Aoki-Rolewicz theorem ([30], Theorem 1.3) provides a $p>0$ such that $\|\cdot\|_{\mathscr{B}_{s}}^{p}$ satisfies the triangle inequality. Since $\|\cdot\|_{\mathscr{B}_{f}^{1 / p}}$ is a quasi-norm, using the Aoki-Rolewicz theorem again, we have a $0<\rho<p$ and $C>0$ such that, for any $\left\{F_{i}\right\}_{i \in \mathrm{N}} \subset \mathscr{B}$ and for any finite subset $I \subset \mathrm{N},\left\|\left(\sum_{i \in I}\left\|F_{i}\right\|_{\mathscr{B}_{s}}^{p}\right)^{1 / p}\right\|_{\mathscr{B}_{f}} \leq C\left(\sum_{i \in I}\left\|F_{i}\right\|_{\mathscr{B}}^{\rho}\right)^{1 / \rho}$ (see [42], Lemma 6).

As $\|\cdot\|_{\mathscr{B}_{f}}$ is a regular quasi-norm, item (4) of Definition 1.1 and the inequality $\sum_{i \in \mathrm{N}}\left\|F_{i}\right\|_{\mathscr{B}}^{\rho}<\infty$ imply that $\left(\sum_{i \in \mathrm{N}}\left\|F_{i}\right\|_{\mathscr{B}_{s}}^{p}\right)^{1 / p}$ is well defined in $\mathscr{B}_{f}$. Since $\mathscr{B}_{f} \subseteq \mathscr{M}_{0}\left(\mathrm{R}^{n}\right)$, we have $\sum_{i=0}^{\infty}\left\|F_{i}(x)\right\|_{\mathscr{B}_{s}}^{p}<\infty$ almost everywhere. Similarly, in view of the fact that $\mathscr{B}_{S}$ is a regular sequence space and $\|\cdot\|_{\mathscr{B}_{s}}^{p}$ is sub-additive, $F(x)=\sum_{i=0}^{\infty} F_{i}(x)$ is well defined a.e. and satisfies $\|F\|_{\mathscr{B}}^{\rho} \leq\left\|\left(\sum_{i=0}^{\infty}\left\|F_{i}\right\|_{\mathscr{B}_{s}}^{p}\right)^{1 / p}\right\|_{\mathscr{B}_{f}}^{\rho} \leq C^{\rho} \sum_{i=0}^{\infty}\left\|F_{i}\right\|_{\mathscr{B}}^{\rho}<\infty$. 
THEOREM 1.7. If $\mathscr{B}_{s}$ is a regular sequence space and $\mathscr{B}_{f}$ is a regular function space, then $\mathscr{B}$ is a quasi-Banach lattice.

Proof. Let $\rho$ be the constant given by Lemma 1.6. Suppose that $G_{i}=$ $\left\{g_{i, j}\right\}_{j \in \mathrm{Z}}, i \in \mathrm{N}$, is a Cauchy sequence in $\mathscr{B}$. There exists a subsequence of $G_{i}$, namely $\mathscr{G}_{i}$ with $\mathscr{G}_{-1}=0$, such that $\sum_{i=0}^{\infty}\left(\left\|\mathscr{G}_{i}-\mathscr{G}_{i-1}\right\|_{\mathscr{B}}\right)^{\rho}<\infty$,

Applying Lemma 1.6, we assert that $\sum_{i=0}^{\infty}\left(\mathscr{G}_{i}-\mathscr{G}_{i-1}\right) \in \mathscr{B}$. Due to the fact that $\mathscr{B}_{f} \subseteq \mathscr{M}_{0}\left(\mathrm{R}^{n}\right)$, the limit function $\lim _{i \rightarrow \infty} \mathscr{G}_{i}=G$ exists a.e. and satisfies $|G| \leq \sum_{j=1}^{\infty}\left|\mathscr{G}_{i}-\mathscr{G}_{i-1}\right| \in \mathscr{B}$. As $\mathscr{B}$ satisfies (1.1), we have $G \in \mathscr{B}$ and $\lim _{l \rightarrow 0}\left\|G-\mathscr{G}_{l}\right\|_{\mathscr{B}} \leq C \lim _{l \rightarrow 0}\left(\sum_{i=l+1}^{\infty}\left\|\mathscr{G}_{i}-\mathscr{G}_{i-1}\right\|_{\mathscr{B}}^{\rho}\right)^{1 / \rho}=0$.

Let $0<a<\infty, \mathscr{B}_{s}$ be a regular sequence space and $\mathscr{B}_{f}$ be a regular function space. Define the quasi-Banach lattice $\mathscr{B}^{1 / a}$ as

$\mathscr{B}^{1 / a}=\left\{\left\{f_{i}\right\}_{i \in \mathrm{Z}}: f_{i} \in \mathscr{M}_{0}\left(\mathrm{R}^{n}\right),\left\{f_{i}\right\}_{i \in \mathrm{Z}} \in \mathscr{B}_{s}^{1 / a}\right.$ a.e. and $\left.\left\|\left\{f_{i}\right\}_{i \in \mathrm{Z}}\right\|_{\mathscr{B}_{s}^{1 / a}} \in \mathscr{B}_{f}^{1 / a}\right\}$

and the quasi-norm of $\mathscr{B}^{1 / a}$ is given by $\|f\|_{\mathscr{B}^{1 / a}}=\|\|\left\{f_{i}\right\}_{i \in Z}\left\|_{\mathscr{B}_{s}^{1 / a}}\right\|_{\mathscr{B}_{f}^{1 / a}}, f=$ $\left\{f_{i}\right\}_{i \in Z}$. For any family of locally integrable functions $f=\left\{f_{i}\right\}_{i \in Z}$, write $|f|^{a}=\left\{\left|f_{i}\right|^{a}\right\}_{i \in \mathrm{Z}}$. We have $\left\||f|^{a}\right\|_{\mathscr{B}^{1 / a}}=\|f\|_{\mathscr{B}}^{a}$.

Let $\mathrm{M}$ denote the Hardy-Littlewood maximal operator. For any sequence of locally integrable functions $f=\left\{f_{i}\right\}_{i \in \mathrm{Z}}$, let $\mathbf{M}(f)=\left\{\mathbf{M}\left(f_{i}\right)\right\}_{i \in \mathbf{Z}}$. We are ready to introduce the admissibility condition for which we can use the pair $\left(\mathscr{B}_{f}, \mathscr{B}_{s}\right)$ to define and study the Littlewood-Paley spaces.

Definition 1.8. Let $0<a \leq 1, \mathscr{B}_{s}$ be a regular sequence space and $\mathscr{B}_{f}$ be a regular function space. The pair $\left(\mathscr{B}_{s}, \mathscr{B}_{f}\right)$ is called an a-admissible pair if there exists a constant $C>0$ such that

$$
\|\mathrm{M}(f)\|_{\mathscr{B}^{1 / a}} \leq C\|f\|_{\mathscr{B}^{1 / a}}
$$

for any $f=\left\{f_{i}\right\}_{i \in Z} \in \mathscr{B}^{1 / a}$. We call $\left(\mathscr{B}_{s}, \mathscr{B}_{f}\right)$ an admissible pair if it is 1-admissible.

The admissibility condition (1.2) can be viewed as the Fefferman-Stein vector-valued maximal inequality on the pair of quasi-Banach spaces $\left(\mathscr{B}_{s}^{1 / a}\right.$, $\left.\mathscr{B}_{f}^{1 / a}\right)$.

Definition 1.9. For $0<a \leq 1$ and any locally integrable function $g$, define the operator $\mathrm{M}_{a}$ as $\left.\mathrm{M}_{a}(g)=\left[\mathrm{M}\left(|g|^{a}\right)\right)\right]^{1 / a}$. Furthermore, for any family of locally integrable functions, $f=\left\{f_{i}\right\}_{i \in Z}$, the operator $\mathbf{M}_{a}$ is defined as $\mathbf{M}_{a}(f)=\left\{\mathbf{M}_{a}\left(f_{i}\right)\right\}_{i \in \mathbf{Z}}$.

The following theorem is a straightforward consequence of the definition of $\mathrm{M}_{a}$. 
THEOREM 1.10. Let $0<a \leq 1, \mathscr{B}_{s}$ be a regular sequence space and $\mathscr{B}_{f}$ be a regular function space. The pair $\mathscr{B}=\left(\mathscr{B}_{s}, \mathscr{B}_{f}\right)$ is a-admissible if and only if $\mathrm{M}_{a}$ is bounded on $\mathscr{B}$.

\section{Littlewood-Paley Spaces}

In this section, we define and study the Littlewood-Paley spaces. Let $\hat{f}$ denote the Fourier transform of $f \in \mathscr{S}^{\prime}\left(\mathrm{R}^{n}\right)$.

Definition 2.1. Let $\alpha \in \mathrm{R}, \mathscr{B}_{s}$ be a regular sequence space and $\mathscr{B}_{f}$ be a regular function space. The Littlewood-Paley space $\dot{F}_{\mathscr{B}_{s}, \mathscr{B}_{f}}^{\alpha}$ consists of those $f \in \mathscr{S}^{\prime}\left(\mathrm{R}^{n}\right) / \mathscr{P}$ satisfying

$$
\|f\|_{\dot{F}_{\mathscr{P}_{S}, \mathscr{O}_{f}}^{\alpha}}=\left\|\left\{2^{j \alpha}\left|f * \varphi_{j}\right|\right\}_{j \in Z}\right\|_{\mathscr{B}}<\infty,
$$

where $\varphi_{j}(x)=2^{j n} \varphi\left(2^{j} x\right)$ and $\varphi \in \mathscr{S}\left(\mathrm{R}^{n}\right)$ satisfies

$$
\operatorname{supp} \hat{\varphi} \subseteq\left\{x \in \mathrm{R}^{n}: 1 / 2 \leq|x| \leq 2\right\} \quad \text { and } \quad|\hat{\varphi}(\xi)| \geq C,
$$

$3 / 5 \leq|x| \leq 5 / 3$ for some $C>0$.

The inhomogeneous Littlewood-Paley space $F_{\mathscr{B}_{s}, \mathscr{B}_{f}}^{\alpha}$ is defined by some standard modifications of the above definition. The results for $F_{\mathscr{B}_{s}, \mathscr{B}_{f}}^{\alpha}$ follow from the corresponding results for $\dot{F}_{\mathscr{B}_{s}, \mathscr{B}_{f}}^{\alpha}$ with some obvious amendments. Thus, in what follows, we present and prove the results for $\dot{F}_{\mathscr{B}_{s}, \mathscr{B}_{f}}^{\alpha}$ only.

Proposition 2.2. Let $\alpha \in \mathrm{R}, \mathscr{B}_{s}$ be a regular sequence space and $\mathscr{B}_{f}$ be a regular function space. We have the continuous embedding $\mathscr{S}_{0}\left(\mathrm{R}^{n}\right) \hookrightarrow \dot{F}_{\mathscr{B}_{s}, \mathscr{B}_{f}}^{\alpha}$.

Proof. Let $\varphi \in \mathscr{S}\left(\mathrm{R}^{n}\right)$ satisfy (2.2). For any $g \in \mathscr{S}_{0}\left(\mathrm{R}^{n}\right)$ and $\theta>0$, by Lemma B.1 of [23], there exists a constant $C>0$ depending on a semi-norm of $g$ on $\mathscr{S}\left(\mathrm{R}^{n}\right)$ such that when $j \geq 0$, we have $\left|\left(\varphi_{j} * g\right)(x)\right| \leq C 2^{-(\theta+\alpha) j}(1+$ $|x|)^{-\kappa}$, and $\left|\left(\varphi_{j} * g\right)(x)\right| \leq C 2^{(\theta+\alpha+\kappa) j}\left(1+2^{j}|x|\right)^{-\kappa} \leq C 2^{-(\theta+\alpha)|j|}(1+|x|)^{-\kappa}$ when $j<0$ where $\kappa$ is given in Definition 1.2. As $(1+|x|)^{-\kappa} \in \mathscr{B}_{f}$, it remains to show that for any $\beta>0,\left\{2^{-\beta|j|}\right\}_{j \in Z} \in \mathscr{B}_{s}$. Using the Aoki-Rolewicz theorem on the quasi-norm $\|\cdot\|_{\mathscr{B}_{s}}$ ([30], Theorem 1.3), we have a $\rho>0$ such that $\left\|\left\{2^{-\beta|j|} \mid\right\}_{j \in Z}\right\|_{\mathscr{B}_{s}}^{\rho} \leq \sum_{j \in Z} 2^{-\beta|j| \rho}\left\|\left\{\delta_{i j}\right\}_{i \in Z}\right\|_{\mathscr{B}_{s}}^{\rho}$, where $\delta_{i j}=1$ when $i=j$ and $\delta_{i j}=0$ when $i \neq j$. Condition (1) in Definition 1.3 assures that $\left\|\left\{\delta_{i j}\right\}_{i \in Z}\right\|_{\mathscr{B}_{s}}$ is independent of $j \in \mathrm{Z}$. Hence, $g \in \dot{F}_{\mathscr{B}_{s}, \mathscr{B}_{f}}^{\alpha}$.

Let $\mathscr{Q}=\left\{Q_{i, k}: i \in \mathrm{Z}, k \in \mathrm{Z}^{n}\right\}$ denote the set of dyadic cubes, where $Q_{i, k}=\left\{\left(x_{1}, \ldots, x_{n}\right) \in \mathrm{R}^{n}: k_{j} \leq 2^{i} x_{j}<k_{j}+1, j=1, \ldots, n\right\}$ and $k=$ $\left(k_{1}, \ldots, k_{n}\right)$. We denote the Lebesgue measure of $Q \in \mathscr{Q}$ by $|Q|$ and the side length of $Q$ by $l(Q)$. 
Definition 2.3. Let $\alpha \in \mathrm{R}, \mathscr{B}_{s}$ be a regular sequence space and $\mathscr{B}_{f}$ be a regular function space. The sequence space $\dot{f}_{\mathscr{B}_{s}, \mathscr{B}_{f}}^{\alpha}$ is the collection of all complex-valued sequences $s=\left\{s_{Q}\right\}_{Q \in \mathscr{Q}}$ such that

$$
\|s\|_{\dot{f}_{\mathscr{P}_{S}, \mathscr{B}_{f}}^{\alpha}}=\left\|\left\{\sum_{|Q|=2^{-j n}}\left(|Q|^{-\alpha / n}\left|s_{Q}\right| \tilde{\chi}_{Q}\right)\right\}_{j \in \mathrm{Z}}\right\|_{\mathscr{B}}<\infty,
$$

where $\tilde{\chi}_{Q}=|Q|^{-1 / 2} \chi_{Q}$.

We define the notion of absolutely continuous quasi-norm (see [5], Chapter 1 , Section 3 ). The absolutely continuity plays a decisive role in the denseness of $\mathscr{S}_{0}\left(\mathrm{R}^{n}\right)$ in $\dot{F}_{\mathscr{B}_{s}, \mathscr{B}_{f}}^{\alpha}$.

Definition 2.4. We say that a quasi-Banach space $B \subset \mathcal{M}(\mu)$ has absolutely continuous quasi-norm if $\lim _{i \rightarrow \infty}\left\|f_{i}\right\|_{B}=0$ for every sequence $\left\{f_{i}\right\}_{i \in \mathrm{N}} \subset B$ satisfying $f_{i} \downarrow 0 \mu$-almost everywhere.

With the preceding preparations, we show that $\dot{f}_{\mathscr{B}_{s}, \mathscr{B}_{f}}^{\alpha}$ is a quasi-Banach lattice and the set of finite sequence is a dense subset of $\dot{f}_{\mathscr{B}_{s}, \mathscr{B}_{f}}^{\alpha}$ provided that $\mathscr{B}_{s}$ and $\mathscr{B}_{f}$ have absolutely continuous quasi-norms.

THEOREM 2.5. Let $\alpha \in \mathrm{R}, \mathscr{B}_{s}$ be a regular sequence space and $\mathscr{B}_{f}$ be a regular function space. The sequence space $\dot{f}_{\mathscr{B}_{s}, \mathscr{B}_{f}}^{\alpha}$ is a quasi-Banach lattice. If $\mathscr{B}_{s}$ and $\mathscr{B}_{f}$ have absolutely continuous quasi-norms, then the set $\mathscr{F}=\{s=$ $\left\{s_{Q}\right\}_{Q \in \mathscr{Q}}$ : only a finitely number of $\left.s_{Q} \neq 0\right\}$ is dense in $\dot{f}_{\mathscr{B}_{s}, \mathscr{B}_{f}}^{\alpha}$.

Proof. We first prove the completeness of $\dot{f}_{\mathscr{B}_{s}, \mathscr{B}_{f}}^{\alpha}$. Let $\rho$ be the constant given by Lemma 1.6. For any Cauchy sequence $c_{i}=\left\{c_{i, Q}\right\}_{Q \in \mathcal{Q}}, i \in \mathrm{N}$, in $\dot{f}_{\mathscr{B}_{s}, \mathscr{B}_{f}}^{\alpha}$, we can assume that $s_{i}=c_{i}-c_{i-1} \in \dot{f}_{\mathscr{B}_{s}, \mathscr{B}_{f}}^{\alpha}$ with $c_{-1}=0$ and $s_{i}=\left\{s_{i, Q}\right\}_{Q \in \mathcal{Q}}, i \in \mathrm{N}$, satisfy $\sum_{i=0}^{\infty}\left\|s_{i}\right\|_{\dot{f}_{\mathscr{B}_{s}, \mathscr{B}_{f}}^{\alpha}}^{\rho}<\infty$. Therefore, $S_{i}(x)=$ $\left\{\sum_{|Q|=2^{-j n}}\left(|Q|^{-\alpha / n}\left|s_{i, Q}\right| \tilde{\chi}_{Q}\right)\right\}_{j \in \mathrm{Z}}, i \in \mathrm{N}$, satisfy $\sum_{i=0}^{\infty}\left\|S_{i}\right\|_{\mathscr{B}}^{\rho}=\sum_{i=0}^{\infty}\left\|s_{i}\right\|_{\dot{f}_{\mathscr{S}_{s}, \mathscr{B}_{f}}^{\alpha}}^{\rho}$ $<\infty$. Lemma 1.6 assures that $S_{\infty}=\sum_{i=0}^{\infty} S_{i}$ exists in $\mathscr{B}$. As $Q_{1}, Q_{2} \in \mathscr{Q}$ with $\left|Q_{1}\right|=\left|Q_{2}\right|$ are either disjoint or identical, we find that

$$
\begin{aligned}
S_{\infty} & =\left\{\sum_{i=0}^{\infty} \sum_{|Q|=2^{-j n}}\left(|Q|^{-\alpha / n}\left|s_{i, Q}\right| \tilde{\chi}_{Q}\right)\right\}_{j \in Z} \\
& =\left\{\sum_{|Q|=2^{-j n}}\left(|Q|^{-\alpha / n}\left(\sum_{i=0}^{\infty}\left|s_{i, Q}\right|\right) \tilde{\chi}_{Q}\right)\right\}_{j \in Z} .
\end{aligned}
$$

Since $\mathscr{B}_{f} \subseteq \mathscr{M}_{0}\left(\mathrm{R}^{n}\right)$, for any $j \in \mathrm{Z}$, the function $\sum_{|Q|=2^{-j n}}\left(|Q|^{-\alpha / n}\left(\sum_{i=0}^{\infty}\left|s_{i, Q}\right|\right) \tilde{\chi}_{Q}\right)$ is Lebesgue measurable and finite almost 
everywhere. Therefore, for any $Q \in \mathcal{Q}, \sum_{i=0}^{\infty}\left|s_{i, Q}\right|<\infty$. That is, $\sum_{i=0}^{\infty} s_{i, Q}$ is well defined and the limit of $c_{i}$ exists. Let $c=\lim _{i \rightarrow \infty} c_{i}=\left\{\sum_{i=0}^{\infty} s_{i, Q}\right\}_{Q \in \mathscr{Q}}$. We have $\left\|c-c_{l}\right\|_{\dot{f}_{B_{s}, B_{f}}^{\alpha}}=\left\|S_{\infty}-\sum_{0}^{l} S_{l}\right\|_{\mathscr{B}} \leq C\left(\sum_{i=l}^{\infty}\left\|S_{i}\right\|_{\mathscr{B}}^{\rho}\right)^{1 / \rho}$ $=C\left(\sum_{i=l}^{\infty}\left\|s_{i}\right\|_{\dot{f}_{\mathscr{B}_{s}, \mathscr{O}_{f}}^{\alpha}}^{\rho}\right)^{1 / \rho} \rightarrow 0$ as $l \rightarrow \infty$.

Next, we prove that $\mathscr{F}$ is dense in $\dot{f}_{\mathscr{B}_{s}, \mathscr{B}_{f}}^{\alpha}$. First of all, by Definition 1.2, $\chi_{Q} \in \mathscr{B}_{f}$ for any $Q \in \mathscr{2}$. Moreover, Definition 1.3 guarantees that the set of finite sequences is a subset of $\mathscr{B}_{s}$. If $t=\left\{t_{Q}\right\}_{Q \in \mathscr{Q}} \in \mathscr{F}$, then there exist a constant $C>0$, a collection of dyadic cubes $\left\{Q_{l}\right\}_{l=1}^{2^{n}} \subset \mathscr{Q}$ and a finite subset $J \subset \mathrm{Z}$ such that $\sum_{|Q|=2^{-j n}}|Q|^{-\alpha / n}\left|t_{Q}\right| \tilde{\chi}_{Q}(x) \leq C \sum_{l=1}^{2^{n}} \chi_{Q_{l}}$ when $j \in J$ and $\sum_{|Q|=2^{-j n}}|Q|^{-\alpha / n}\left|t_{Q}\right| \tilde{\chi}_{Q}(x)=0$ when $j \in Z \backslash J$. By Definitions 1.1, 1.2 and 1.3, we conclude that $t \in \dot{f}_{\mathscr{B}_{s}, \mathscr{P}_{f}}^{\alpha}$ and, hence, $\mathscr{F} \subset \dot{f}_{\mathscr{B}_{s}, \mathscr{B}_{f}}^{\alpha}$.

Let $s=\left\{s_{Q}\right\}_{Q \in \mathscr{Q}} \in \dot{f}_{\mathscr{B}_{s}, \mathscr{B}_{f}}^{\alpha}$. For any $N \in \mathrm{N}$, consider $s_{N}=\left\{s_{Q}^{N}\right\}_{Q \in \mathscr{Q}} \in \mathscr{F}$ where $s_{Q}^{N}=s_{Q}$ if $\left|x_{Q}\right| \leq N$ and $2^{-N} \leq|Q| \leq 2^{N}$; and $s_{Q}^{N}=0$ otherwise. Write $S^{N}(x)=\left\{\sum_{|Q|=2^{-j n}}\left(|Q|^{-\alpha / n}\left|s_{Q}^{N}\right| \tilde{\chi}_{Q}\right)\right\}_{j \in Z}$ and $S(x)=$ $\left\{\sum_{|Q|=2^{-j n}}\left(|Q|^{-\alpha / n}\left|s_{Q}\right| \tilde{\chi}_{Q}\right)\right\}_{j \in Z}$. We have $0 \leq S-S_{N+1} \leq S-S_{N}$ and $\left(S-S_{N}\right) \downarrow 0$ in $\mathscr{B}_{s}$. As $\mathscr{B}_{S}$ is a quasi-Banach lattice and has absolutely continuous quasi-norm, we have $\left\|S-S_{N}\right\|_{\mathscr{B}_{s}} \downarrow 0$ in $\mathscr{B}_{f}$. Similarly, we find that \|\|$S-S_{N}\left\|_{\mathscr{B}_{s}}\right\|_{\mathscr{B}_{f}} \downarrow 0$ because $\mathscr{B}_{f}$ has absolutely continuous quasi-norm. Thus, $\lim _{N \rightarrow \infty}\left\|s-s_{N}\right\|_{\dot{f}_{\mathscr{B}_{s}, \mathscr{B}_{f}}^{\alpha}}=\lim _{N \rightarrow \infty}\left\|S-S_{N}\right\|_{\mathscr{B}}=\lim _{N \rightarrow \infty}\|\| S-S_{N}\left\|_{\mathscr{B}_{s}}\right\|_{\mathscr{B}_{f}}=$ 0 .

\section{The Frazier-Jawerth theory for $\dot{\boldsymbol{F}}_{\mathscr{S}_{s}, \mathscr{B}_{f}}^{\alpha}$}

In this section, we show that the $\varphi-\psi$ transforms [21], [22], [23], [24] provide an association between $\dot{F}_{\mathscr{B}_{s}, \mathscr{B}_{f}}^{\alpha}$ and $\dot{f}_{\mathscr{B}_{s}, \mathscr{B}_{f}}^{\alpha}$. With this connection, we can transfer some results, for instance, the completeness, from $\dot{f}_{\mathscr{B}_{s}, \mathscr{P}_{f}}^{\alpha}$ to $\dot{F}_{\mathscr{B}_{s}, \mathscr{O}_{f}}^{\alpha}$.

Although we follow the ideas from [23], some results in our setting cannot be directly recalled from [23] because there are some techniques in [23], for example, the duality of the Triebel-Lizorkin spaces, which are unavailable in our setting. However, we can derive all of our results by using the admissibility condition (1.2) only.

The $\varphi-\psi$ transforms consist of two operators $\mathrm{S}_{\varphi}$ and $\mathrm{T}_{\psi}$ generated by a pair of functions $\varphi, \psi \in \mathscr{S}\left(\mathrm{R}^{n}\right)$ satisfying (2.2) and $\sum_{j \in \mathrm{Z}} \hat{\varphi}\left(2^{-j} \xi\right) \overline{\hat{\psi}\left(2^{-j} \xi\right)}=1$, $\xi \neq 0$ (see [23], p. 45 (2.1)-(2.4)). We set $\varphi_{v}(x)=2^{v n} \varphi\left(2^{v} x\right), \psi_{v}(x)=$ $2^{v n} \psi\left(2^{v} x\right)$ and $\varphi_{Q}(x)=|Q|^{-1 / 2} \varphi\left(2^{v} x-k\right), \psi_{Q}(x)=|Q|^{-1 / 2} \psi\left(2^{v} x-k\right)$, $v \in \mathrm{Z}, k \in \mathrm{Z}^{n}$ and $Q=Q_{v, k}$.

For any $f \in \mathscr{S}^{\prime}\left(\mathrm{R}^{n}\right) / \mathscr{P}$ and for any complex-valued sequence $s=\left\{s_{Q}\right\}_{Q \in \mathscr{Q}}$, 
we define $\mathrm{S}_{\varphi}(f)=\left\{\left(\mathrm{S}_{\varphi} f\right)_{Q}\right\}_{Q \in \mathcal{Q}}=\left\{\left\langle f, \varphi_{Q}\right\rangle\right\}_{Q \in \mathcal{Q}}$ and $\mathrm{T}_{\psi}(s)=\sum_{Q} s_{Q} \psi_{Q}$. It is well known that $\mathrm{T}_{\psi} \circ \mathrm{S}_{\varphi}=i d$ in $\mathscr{S}^{\prime}\left(\mathrm{R}^{n}\right) / \mathscr{P}$ (see [28], Theorem 6.1).

The following result is the main theorem of this section:

THEOREM 3.1. Let $\alpha \in \mathrm{R}, 0<a \leq 1$ and $\left(\mathscr{B}_{s}, \mathscr{B}_{f}\right)$ be an a-admissible pair. The Littlewood-Paley space $\dot{F}_{\mathscr{P}_{s}}^{\alpha}, \mathscr{B}_{f}$ is independent of the function $\varphi$ in Definition 2.1. The linear operators $\mathrm{S}_{\varphi}: \dot{F}_{\mathscr{P}_{s}, \mathscr{B}_{f}}^{\alpha} \rightarrow \dot{f}_{\mathscr{B}_{s}, \mathscr{B}_{f}}^{\alpha}$ and $\mathrm{T}_{\psi}: \dot{f}_{\mathscr{B}_{s}, \mathscr{B}_{f}}^{\alpha} \rightarrow$ $\dot{F}_{\mathscr{B}_{s}, \mathscr{B}_{f}}^{\alpha}$ are bounded. Moreover, we have constants $C_{1}>C_{2}>0$ such that for any $f \in \dot{F}_{\mathscr{B}_{s}, \mathscr{B}_{f}}^{\alpha}$,

$$
C_{2}\|f\|_{\dot{F}_{\mathscr{S}_{s}, \mathscr{O}_{f}}^{\alpha}} \leq\left\|\mathrm{S}_{\varphi}(f)\right\|_{\dot{f}_{\mathscr{B}_{s}, \mathscr{P}_{f}}^{\alpha}} \leq C_{1}\|f\|_{\dot{F}_{\mathscr{P}_{s}, \mathscr{O}_{f}}^{\alpha}}
$$

Proof. We only sketch the proof for the boundedness of $\mathrm{S}_{\varphi}$. With some simple modifications of Peetre's lemma (see [56] Sections 1.4.1 and 1.4.2), the definitions of $\mathrm{S}_{\varphi}$ and $\mathrm{M}_{a}$ yield $\sum_{|Q|=2^{-j n}}|Q|^{-1 / 2-\alpha / n}\left|\left(\mathrm{~S}_{\varphi} f\right)_{Q}\right| \chi_{Q}(x) \leq$ $C \mathrm{M}_{a}\left(2^{j \alpha}\left(\tilde{\varphi}_{j} * f\right)\right)(x)$ where $\tilde{\varphi}(x)=\overline{\varphi(-x)}$. Therefore,

$$
\begin{aligned}
\left\|\left\{\left(\mathrm{S}_{\varphi} f\right)_{Q}\right\}_{Q}\right\|_{\tilde{f}_{\mathscr{B}_{s}, \mathscr{B}_{f}}^{\alpha}} & =\left\|\left\{\sum_{Q=2^{-j n}}\left(|Q|^{-\alpha / n-1 / 2}\left|\left(\mathrm{~S}_{\varphi} f\right)_{Q}\right| \chi_{Q}(x)\right)\right\}_{j \in \mathrm{Z}}\right\|_{\mathscr{B}} \\
& \leq C\left\|\left\{\mathrm{M}_{a}\left(2^{j \alpha}\left|\tilde{\varphi}_{j} * f\right|\right)\right\}_{j \in Z}\right\|_{\mathscr{B}} .
\end{aligned}
$$

The boundedness of $\mathrm{M}_{a}$ asserts the boundedness of $\mathrm{S}_{\varphi}$. The rest of the proof is similar to the proof of [23], Theorem 2.2, therefore, for simplicity, we omit the details.

With the boundedness of the $\varphi-\psi$ transforms, we show that $\dot{F}_{\mathscr{B}_{s}, \mathscr{B}_{f}}^{\alpha}$ is a quasi-Banach space and $\dot{F}_{\mathscr{B}_{s}, \mathscr{B}_{f}}^{\alpha}$ has $\mathscr{S}_{0}\left(\mathrm{R}^{n}\right)$ as a dense subset.

Theorem 3.2. Let $\alpha \in \mathrm{R}, 0<a \leq 1$ and $\left(\mathscr{B}_{s}, \mathscr{B}_{f}\right)$ be an a-admissible pair. The Littlewood-Paley space $\dot{F}_{\mathscr{B}_{s}, \mathscr{B}_{f}}^{\alpha}$ is a quasi-Banach space. If $\mathscr{B}_{s}$ and $\mathscr{B}_{f}$ have absolutely continuous quasi-norms, then $\mathscr{S}_{0}\left(\mathrm{R}^{n}\right)$ is dense in $\dot{F}_{\mathscr{B}_{s}, \mathscr{B}_{f}}^{\alpha}$.

Proof. Let $F_{i}, i \in \mathrm{N}$, be a Cauchy sequence in $\dot{F}_{\mathscr{B}_{s}, \mathscr{B}_{f}}^{\alpha}$. By Theorem 3.1, $\left\{\left(\mathrm{S}_{\varphi} F_{i}\right)_{Q}\right\}_{Q \in \mathscr{Q}}$ is a Cauchy sequence in $\dot{f}_{\mathscr{B}_{s}, \mathscr{B}_{f}}^{\alpha}$. Hence, $\left\{\left(\mathrm{S}_{\varphi} F_{i}\right)_{Q}\right\}_{Q \in \mathscr{Q}}$ converges to $s=\left\{s_{Q}\right\}_{Q \in \mathscr{Q}}$ in $\dot{f}_{\mathscr{B}_{s}, \mathscr{B}_{f}}^{\alpha}$. Define $F=\mathrm{T}_{\psi} s$. Thus, by Theorem 3.1, $F \in \dot{F}_{\mathscr{P}_{s}, \mathscr{B}_{f}}^{\alpha}$ and $\left\|F-F_{i}\right\|_{\dot{F}_{\mathscr{O}_{s}, \mathscr{B}_{f}}^{\alpha}}=\left\|\mathrm{T}_{\psi} s-\left(\mathrm{T}_{\psi} \circ \mathrm{S}_{\varphi}\right)\left(F_{i}\right)\right\|_{\dot{F}_{\mathscr{P}_{s}, \mathscr{O}_{f}}} \leq \| s-$ $\mathrm{S}_{\varphi} F_{i} \|_{f_{\mathscr{S}_{S}, \mathscr{f}_{f}}^{\alpha}} \rightarrow 0$, as $i \rightarrow \infty$. Hence, $F$ is the limit of the Cauchy sequence $F_{i}, i \in \mathrm{N}$, in $\dot{F}_{\mathscr{O}_{s}, \mathscr{B}_{f}}^{\alpha}$. Moreover, $\mathscr{S}_{0}\left(\mathrm{R}^{n}\right)$ is dense in $\dot{F}_{\mathscr{B}_{s}, \mathscr{B}_{f}}^{\alpha}$ because of Proposition 2.2, Theorem 2.5, Theorem 3.1 and the fact that $\psi_{Q} \in \mathscr{S}_{0}\left(\mathrm{R}^{n}\right)$ for any $Q \in \mathcal{Q}$. 
We find that for any $\varphi \in \mathscr{S}\left(\mathrm{R}^{n}\right)$ satisfying (2.2), the inverse Fourier transform of the functions $|\xi|^{2} \hat{\varphi}(\xi)$ and $|\xi|^{-2} \hat{\varphi}(\xi)$ also satisfy (2.2), therefore, Theorem 3.1 yields the following result.

THeOREM 3.3. Let $\alpha \in \mathrm{R}, 0<a \leq 1$ and $\left(\mathscr{B}_{s}, \mathscr{B}_{f}\right)$ be an a-admissible pair. The Laplacian $\triangle$ is a linear topological isomorphism from $\dot{F}_{\mathscr{B}_{s}, \mathscr{B}_{f}}^{\alpha}$ to $\dot{F}_{\mathscr{B}_{s}, \mathscr{B}_{f}}^{\alpha-2}$.

The Littlewood-Paley spaces possess smooth atomic decompositions and smooth molecular decompositions (for the corresponding results for TriebelLizorkin spaces, see [23], Theorems 3.5, 3.7 and 4.1).

Smooth atomic and molecular decompositions for the Littlewood-Paley spaces can be obtained by following the corresponding arguments for the Triebel-Lizorkin spaces as given in [23], Theorems 3.5, 3.7 and 4.1. We state a lemma used to establish the smooth molecular decompositions for the Littlewood-Paley spaces.

Lemma 3.4. Let $0<a \leq 1$ and $\left(\mathscr{B}_{s}, \mathscr{B}_{f}\right)$ be an a-admissible pair. For any $\epsilon>0$, the linear operators

$$
T_{1}\left(\left\{a_{i}\right\}_{i \in \mathrm{Z}}\right)=\left\{\sum_{j \leq i} 2^{(j-i) \epsilon} a_{j}\right\}_{i \in \mathrm{Z}} \text { and } T_{2}\left(\left\{a_{i}\right\}_{i \in \mathrm{Z}}\right)=\left\{\sum_{j>i} 2^{(i-j) \epsilon} a_{j}\right\}_{i \in \mathrm{Z}}
$$

are bounded on $\mathscr{B}_{s}^{1 / a}$.

The above lemma can be proved by using the Aoki-Rolewicz theorem ([30], Theorem 1.3) and Definition 1.3, item (1).

The proof of the smooth molecular decompositions relies on the boundedness of the almost diagonal operators. We now recall the definition of almost diagonal operators from [23] p. 53, (3.1).

Definition 3.5. Let $0 \leq a \leq 1, \alpha \in \mathrm{R}$ and $\left(\mathscr{B}_{s}, \mathscr{B}_{f}\right)$ be an $a$-admissible pair. Let $J=\max (n, n / a)$. The matrix $A=\left\{a_{Q P}\right\}_{P, Q \in \mathscr{Q}}$ is an almost diagonal operator for $\dot{f}_{\mathscr{B}_{s}, \mathscr{B}_{f}}^{\alpha}$ if there exists $\epsilon>0$ such that

$$
\sup _{Q, P}\left|a_{Q P}\right| / \omega_{Q P}(\epsilon)<\infty,
$$

where

$$
\begin{aligned}
\omega_{Q P}(\epsilon)=\left(\frac{l(Q)}{l(P)}\right)^{\alpha}(1+ & \left.\frac{\left|x_{Q}-x_{P}\right|}{\max (l(P), l(Q))}\right)^{-J-\epsilon} \\
& \min \left[\left(\frac{l(Q)}{l(P)}\right)^{(n+\epsilon) / 2},\left(\frac{l(P)}{l(Q)}\right)^{(n+\epsilon) / 2+J-n}\right] .
\end{aligned}
$$


The proof of the following theorem is based on the method from Theorem 3.3 of [23] but some ideas in [23] can no longer be used in this paper. Even though we lack of those special techniques in [23], we manage to obtain our results by solely using the admissibility condition (1.2). This is the reason why we only assign (1.2) as the admissibility condition for our study.

THeOREM 3.6. Let $0<a \leq 1, \alpha \in \mathrm{R}$ and $\left(\mathscr{B}_{s}, \mathscr{B}_{f}\right)$ be an a-admissible pair. An almost diagonal operator for $\dot{f}_{\mathscr{B}_{s}, \mathscr{B}_{f}}^{\alpha}$ is a bounded linear operator on $\dot{f}_{\mathscr{B}_{s}, \mathscr{B}_{f}}^{\alpha}$.

Proof. Let $A=\left\{a_{Q P}\right\}_{P, Q \in \mathcal{Q}}$ be an almost diagonal operator for $\dot{f}_{\mathscr{S}_{s}, \mathscr{B}_{f}}^{\alpha}$. Let $\left(A_{0} s\right)_{Q}=\sum_{l(Q) \leq l(P)} a_{Q P} s_{P}$ and $\left(A_{1} s\right)_{Q}=\sum_{l(Q)>l(P)} a_{Q P} s_{P}$, for $\left\{s_{Q}\right\}_{Q \in \mathcal{Q}} \in$ $\dot{f}_{\mathscr{B}_{s}, \mathscr{B}_{f}}^{\alpha}$. Let $l(Q)=2^{-i}$. By [23] Lemma A.2 and Remark A.3, we have

$$
\begin{aligned}
& \sum_{l(Q)=2^{-i}}|Q|^{-\alpha / n}\left|\left(A_{1} s\right)_{Q}\right| \tilde{\chi}_{Q}(x) \\
& \leq C \sum_{j>i} 2^{(i-j) \epsilon / 2} \mathrm{M}_{a}\left(\sum_{l(P)=2^{-j}}|P|^{-\alpha / n}\left|s_{P}\right| \tilde{\chi}_{P}\right)(x) .
\end{aligned}
$$

Hence,

$$
\left\|A_{1} s\right\|_{\dot{f}_{\mathscr{B}_{s}, \mathscr{B}_{f}}^{\alpha}} \leq C\left\|\left\{\sum_{j>i} 2^{(i-j) a \epsilon / 2}\left(\mathrm{M}_{a}\left(\sum_{|P|=2^{-j n}}|P|^{-\alpha / n}\left|s_{P}\right| \tilde{\chi}_{P}\right)\right)^{a}\right\}_{i \in Z}\right\|_{\mathscr{B}^{1 / a}}^{1 / a}
$$

by the definition of $\mathscr{B}^{1 / a}$. As $T_{2}$ is bounded, we deduce that

$$
\left\|A_{1} s\right\|_{\tilde{f}_{\mathscr{S}_{s}, \mathscr{O}_{f}}^{\alpha}} \leq C\left\|\left\{\left(\mathrm{M}_{a}\left(\sum_{|P|=2^{-j n}}|P|^{-\alpha / n}\left|s_{P}\right| \tilde{\chi}_{P}\right)\right)^{a}\right\}_{j \in \mathrm{Z}}\right\|_{\mathscr{B}^{1 / a}}^{1 / a} .
$$

Moreover, as $\left(\mathscr{B}_{s}, \mathscr{B}_{f}\right)$ is $a$-admissible and $\left(\mathrm{M}_{a}(f)\right)^{a}=\mathrm{M}\left(|f|^{a}\right)$, we obtain $\left\|A_{1} s\right\|_{\dot{f}_{\mathscr{S}_{s}, \mathscr{O}_{f}}^{\alpha}} \leq C\|s\|_{\dot{f}_{\mathscr{S}_{s}, \mathscr{O}_{f}}^{\alpha}}$. We apply the same method to estimate $A_{0}$. Hence, $A=A_{0}+A_{1}$ is bounded on $\dot{f}_{\mathscr{B}_{s}, \mathscr{B}_{f}}^{\alpha}$.

Smooth molecular decompositions for $\dot{F}_{\mathscr{B}_{s}, \mathscr{B}_{f}}^{\alpha}$ can be established provided that $\left(\mathscr{B}_{s}, \mathscr{B}_{f}\right)$ is an $a$-admissible pair for some $0<a \leq 1$. For brevity, we skip the proof and the reader is referred to [23], Theorems 3.5 and 3.7 for details. 


\section{Rearrangement-invariant quasi-Banach function spaces}

In the rest of this paper, we apply our general theory to some important function spaces. We begin with the r.-i. quasi-Banach function space $X$. The r.-i. quasi-Banach function spaces include a significant number of function spaces appeared in analysis, for instance, the Lorentz spaces and the Orlicz spaces. The main results of this section are Theorem 4.8 which offers the conditions on $X$ for the admissibility of the pair $\left(\mathscr{B}_{s}, X\right)$ and Theorem 4.10 which shows the Littlewood-Paley characterization of $X$. With the Littlewood-Paley characterization of $X$, we can determine the condition for which r.-i. quasi-Banach function spaces belong to the Littlewood-Paley spaces.

For any $f \in \mathscr{M}\left(\mathrm{R}^{n}\right)$, let $f^{*}$ denote the decreasing rearrangement of $f$.

Definition 4.1. A quasi-Banach space $X \subset \mathcal{M}\left(\mathrm{R}^{n}\right)$ is called a $r$-i. quasiBanach function space if there exists a regular quasi-norm $\rho_{X}: \mathscr{M}([0, \infty)) \rightarrow$ $[0, \infty]$ so that $\|f\|_{X}=\rho_{X}\left(f^{*}\right), f \in X$, where $\mathcal{M}([0, \infty))$ is the set of Lebesgue measurable functions on $[0, \infty)$. That is, the r.-i. quasi-Banach function space $X$ has the Luxemburg type representation (see [5], Chapter 2, Theorem 4.10).

If $X$ is a r.-i. Banach function space, the Luxemburg representation of $X$ arises from an integral formula related to the associated space of $X$. On the other hand, a r.-i. quasi-Banach function space does not necessarily have non-trivial associate space. Hence, we cannot rewrite the norm $\|\cdot\|_{X}$ as the supremum of some appropriate integrals (see [36], Volume II, p. 146-147 or [5], Chapter 3, proof of Theorem 5.15). Therefore, the Luxemburg type representation gives us an access to express the norm of $f$ in term of $f^{*}$. In Theorem 4.4, we demonstrate the use of the Luxemburg type representation in the proof of the boundedness of quasi-linear operators of joint weak type.

For any $s \geq 0$ and $f \in \mathscr{M}\left(\mathrm{R}^{n}\right)$, define $\left(D_{s} f\right)(x)=f(s x), x \in \mathrm{R}^{n}$. Let $\left\|D_{s}\right\|_{X \rightarrow X}$ be the operator norm of $D_{s}$ on $X$. We recall the definition of Boyd's indices for r.-i. quasi-Banach function spaces from [42].

Definition 4.2. Let $X$ be a r.-i. quasi-Banach function space on $\mathrm{R}^{n}$. Define the lower Boyd index of $X, p_{X}$, and the upper Boyd index of $X, q_{X}$, by

$$
p_{X}=\sup \left\{p: \exists C>0 \text { such that } \forall 0 \leq s<1,\left\|D_{s}\right\|_{X \rightarrow X} \leq C s^{-n / p}\right\}
$$

and

$$
q_{X}=\inf \left\{q: \exists C>0 \text { such that } \forall 1 \leq s,\left\|D_{s}\right\|_{X \rightarrow X} \leq C s^{-n / q}\right\},
$$

respectively. 
We have $0 \leq p_{X} \leq q_{X} \leq \infty$. For example, the Boyd indices of the Lebesgue spaces $L^{p}, 0<p \leq \infty$, with the quasi-norm $\|f\|_{L^{p}}=\left(\int|f(x)|^{p} d x\right)^{1 / p}$ are $p_{L^{p}}=q_{L^{p}}=p$.

We now establish the Fefferman-Stein type vector-valued maximal inequalities on $X$. We need some notations and preliminary results.

Let $X$ be a r.-i. quasi-Banach function space. Recall that for any $p \in(0, \infty)$, $X^{p}$ is the $1 / p$-th power of $X$. Obviously, $X^{p}$ is a r.-i. quasi-Banach function space. According to Definition 4.2, we find that for any $p \in(0, \infty), p_{X^{p}}=$ $p p_{X}$ and $q_{X^{p}}=p q_{X}$.

Next, we state the definition of the $B$-valued quasi-Banach function spaces where $B$ is a separable Banach space. Let $\mathscr{M}_{0}\left(\mathrm{R}^{n}, B\right)=\left\{f: \mathrm{R}^{n} \rightarrow B\right.$ : $\left.L(f) \in \mathcal{M}_{0}\left(\mathrm{R}^{n}\right), \forall L \in B^{*}\right\}$ where $B^{*}$ is the dual space of $B$. Let $\|f\|_{B}^{*}(t)$ be the decreasing rearrangement of $\|f(x)\|_{B}$. Given a quasi-Banach function space on $\mathrm{R}^{n}, X$, and a separable Banach space $B$, let

$$
X_{B}=\left\{f: \mathrm{R}^{n} \rightarrow B: L(f) \in \mathscr{M}\left(\mathrm{R}^{n}\right), \forall L \in B^{*} \text { and }\|f\|_{B} \in X\right\} .
$$

The vector space $X_{B}$ is endowed with the quasi-norm $\|\cdot\|_{X_{B}}=\|\| \cdot\left\|_{B}\right\|_{X}=$ $\rho_{X}\left(\|\cdot\|_{B}^{*}\right)$. It is evident that $X_{B}$ is a quasi-Banach space (see Theorem 1.7).

Let $B_{0}$ and $B_{1}$ be separable Banach spaces. A mapping $T: E \rightarrow \mathscr{M}_{0}\left(\mathrm{R}^{n}, B_{1}\right)$, where $E$ is a subspace of $\mathscr{M}_{0}\left(\mathrm{R}^{n}, B_{0}\right)$, is a quasi-linear operator if there exists $k \geq 1$ such that for any $f, g \in E$,

$$
\begin{aligned}
& \|T(f+g)\|_{B_{1}} \leq k\left(\|T f\|_{B_{1}}+\|T g\|_{B_{1}}\right) \quad \text { and }\|T(\lambda f)\|_{B_{1}}=|\lambda|\|T f\|_{B_{1}}, \\
& \lambda \in \mathrm{C} .
\end{aligned}
$$

Definition 4.3. Let $1 \leq p, q \leq \infty$ and $B_{0}, B_{1}$ be separable Banach spaces. Write $\sigma=\left(p, q, B_{0}, B_{1}\right)$. A quasi-linear operator $T$ is of joint weak type $\sigma$ if there is a constant $C>0$ such that $\|T(f)\|_{B_{1}}^{*}(t) \leq C S_{\sigma}\left(\|f\|_{B_{0}}^{*}\right)(t)$, $t>0$, where $S_{\sigma}$ is the Calderón operator given by

$$
\left(S_{\sigma} g\right)(t)=t^{-1 / p} \int_{0}^{t} s^{1 / p} g(s) \frac{d s}{s}+t^{-1 / q} \int_{t}^{\infty} s^{1 / q} g(s) \frac{d s}{s}, \quad t>0 .
$$

If $T$ is a bounded quasi-linear operator from $L_{B_{0}}^{p}$ to $L_{B_{1}}^{p}$ and from $L_{B_{0}}^{q}$ to $L_{B_{1}}^{q}$, then $T$ is of joint weak type ( $p, q, B_{0}, B_{1}$ ). The reader may consult [5], pp. 141-154 and pp. 222-226, for the proof of the above assertion and some other basic results for the operators of joint weak type when $B_{0}=B_{1}=\mathrm{C}$. The proof of the above assertion for $B$-valued function spaces relies on the ideas given in [55], Sections 1.18.6 and 1.18.7. We study the boundedness of quasi-linear operators of joint weak type on $X_{B}$. 
Theorem 4.4. Suppose that $1 \leq p<q \leq \infty$. Let $B_{0}$ and $B_{1}$ be separable Banach spaces and $X$ be a r.-i.quasi-Banach function space on $\mathrm{R}^{n}$. If the Boyd indices of $X$ satisfy $p<p_{X} \leq q_{X}<q$, then every quasi-linear operator of joint weak type ( $\left.p, q, B_{0}, B_{1}\right)$ is bounded from $X_{B_{0}}$ to $X_{B_{1}}$.

Proof. Let $T$ be a quasi-linear operator of joint weak type ( $\left.p, q, B_{0}, B_{1}\right)$. By using the substitution $s=u t$, we have

$$
\begin{aligned}
\|T(f)\|_{B_{1}}^{*}(t) & \leq C\left(\int_{0}^{1} u^{1 / p-1}\|f\|_{B_{0}}^{*}(u t) d u+\int_{1}^{\infty} u^{1 / q-1}\|f\|_{B_{0}}^{*}(u t) d u\right) \\
& \leq C\left(\sum_{k=-\infty}^{0} 2^{n k / p}\|f\|_{B_{0}}^{*}\left(2^{n k} t\right)+\sum_{k=0}^{\infty} 2^{n k / q}\|f\|_{B_{0}}^{*}\left(2^{n k} t\right)\right) .
\end{aligned}
$$

Let $\kappa$ be the index given by the Aoki-Rolewicz theorem for the quasi-norm $\rho_{X}$ ([30], Theorem 1.3) so that $\rho_{X}^{\kappa}$ is sub-additive. We find that

$$
\begin{aligned}
& \|T(f)\|_{X_{B_{1}}}^{\kappa} \\
& \quad \leq C\left(\sum_{k=-\infty}^{0} 2^{n k \kappa / p}\left[\rho_{X}\left(\|f\|_{B_{0}}^{*}\left(2^{n k} t\right)\right)\right]^{\kappa}+\sum_{k=0}^{\infty} 2^{n k \kappa / q}\left[\rho_{X}\left(\|f\|_{B_{0}}^{*}\left(2^{n k} t\right)\right)\right]^{\kappa}\right) \\
& \quad=C\left(\sum_{k=-\infty}^{0} 2^{n k \kappa / p}\left[\left\|D_{2^{k}}\right\| f\left\|_{B_{0}}\right\|_{X}\right]^{\kappa}+\sum_{k=0}^{\infty} 2^{n k \kappa / q}\left[\left\|D_{2^{k}}\right\| f\left\|_{B_{0}}\right\|_{X}\right]^{\kappa}\right) .
\end{aligned}
$$

In view of the Luxemburg type representation of $\|\cdot\|_{X}$, we establish the first inequality. We have the last identity because $\left(D_{2^{k}}(f)\right)^{*}=D_{2^{n k}}\left(f^{*}\right)$. According to Definition 4.2, there exist $p<p_{0}<p_{X}$ and $q_{X}<q_{0}<q$ such that

$$
\begin{aligned}
\|T(f)\|_{X_{B_{1}}}^{\kappa} & \leq C\left(\sum_{k=-\infty}^{0} 2^{n k \kappa\left(1 / p-1 / p_{0}\right)}\|f\|_{X_{B_{0}}}^{\kappa}+\sum_{k=0}^{\infty} 2^{n k \kappa\left(1 / q-1 / q_{0}\right)}\|f\|_{X_{B_{0}}}^{\kappa}\right) \\
& \leq C\|f\|_{X_{B_{0}}}^{\kappa} .
\end{aligned}
$$

Our promised result follows obviously from the above inequalities.

We recall the definition of the UMD property.

Definition 4.5. A Banach space $B$ is said to have the UMD property if, for $1<p<\infty$, the martingale difference sequences $d=\left\{d_{i}\right\}_{i \in \mathrm{N}}$ in $L_{B}^{p}([0,1])$ are unconditional; that is,

$$
\left(\int_{0}^{1}\left\|\sum_{i \in \mathrm{N}} \epsilon_{i} d_{i}(x)\right\|_{B}^{p} d x\right)^{1 / p} \leq C_{p, B}\left(\int_{0}^{1}\left\|\sum_{i \in \mathrm{N}} d_{i}(x)\right\|_{B}^{p} d x\right)^{1 / p},
$$


whenever $\epsilon_{i} \in\{1,-1\}$ and $i \in \mathrm{N}$.

Next is one of the important properties for Banach spaces having the UMD property. The proof of the following result is given in [9], Section 2 and [50], Theorem 3.

TheORem 4.6. Let $1<p<\infty$ and $B$ be a Banach lattice of measurable functions in a $\sigma$-finite measure space. If $B$ has the UMD property, then the quasi-linear operator $\mathrm{M}: L_{B}^{p} \rightarrow L_{B}^{p}$ is bounded.

Thus, the quasi-linear operator $\mathrm{M}$ is of joint weak type $(p, q, B, B), 1<$ $p, q<\infty$, when $B$ has the UMD property. Another important feature of the UMD property is given below. For the proof, the reader is referred to [50], Theorem 4.

Theorem 4.7. Let $B$ be a Banach lattice having the UMD property. There exists a $\epsilon_{B}>0$ such that $B^{q}$ has the UMD property when $\frac{1}{1+\epsilon_{B}}<q<\infty$.

We obtain one of the main results of this section.

THeorem 4.8. Let $0<p \leq 1$ and $X$ be a r.-i. quasi-Banach function space on $\mathrm{R}^{n}$ with $0<p_{X} \leq q_{X}<\infty$. Suppose that $B$ is a regular Banach sequence space having the UMD property. The pair $\left(B^{p}, X\right)$ is a-admissible provided that a satisfies $0<a<\min \left(p_{X}, p\left(1+\epsilon_{B}\right)\right)$.

Proof. Let $\rho$ be the constant such that $\|\cdot\|_{X}^{\rho}$ is sub-additive ([30], Theorem 1.3). We see that $\left\|(1+|x|)^{-\kappa}\right\|_{X}^{\rho} \leq \sum_{k \in \mathrm{N}}^{\infty} k^{n}(1+|k|)^{-\kappa \rho}\left\|\chi_{[0,1)^{n}}\right\|_{X}^{\rho}<\infty$ when $\kappa>\frac{n+1}{\rho}$. Thus, $X$ is a regular function space. We consider $X^{1 / a}$ and $B^{p / a}$. We have $1<\frac{p_{X}}{a}=p_{X^{1 / a}} \leq q_{X^{1 / a}}=\frac{q_{X}}{a}<\infty$. Using Theorem 4.7, $B^{p / a}$ has the UMD property. Theorem 4.4 yields

$$
\|\mathrm{M} g\|_{X_{B^{p / a}}^{1 / a}} \leq C\|g\|_{B_{B^{p / a}}^{1 / a}}, \quad \forall g \in X_{B^{p / a}}^{1 / a} .
$$

As $\left(B^{p}\right)^{1 / a}=B^{p / a}$ (see [46], Lemma 2.20(i)), the $a$-admissibility of $\left(B^{p}, X\right)$ follows from (4.1).

We establish the Littlewood-Paley characterization of r.-i. quasi-Banach function spaces. We first present a lemma to overcome the obstacles appearing in the use of the Littlewood-Paley analysis. More precisely, for any $f \in$ $\mathscr{S}^{\prime}\left(\mathrm{R}^{n}\right)$, we have the Littlewood-Paley representation $f=\sum_{v \in Z} f * \varphi_{\nu} * \psi_{v}$ in $\mathscr{S}^{\prime}\left(\mathrm{R}^{n}\right) / \mathscr{P}$ (see [29]), for some $\varphi, \psi \in \mathscr{S}_{0}\left(\mathrm{R}^{n}\right)$. Therefore, in order to use the Littlewood-Paley representation for $f \in X$, we have to show the belonging $X \subset \mathscr{S}^{\prime}\left(\mathrm{R}^{n}\right) / \mathscr{P}$ and the uniqueness of the Littlewood-Paley representation of $f \in X$. 
Lemma 4.9. Let $X$ be a r.-i. quasi-Banach function space on $\mathrm{R}^{n}$. If the upper Boyd index of $X$ satisfies $q_{X}<\infty$, then $X \cap \mathscr{P}=\{0\}$. Moreover, if $1<p_{X} \leq q_{X}<\infty$, then $X \subset \mathscr{S}^{\prime}\left(\mathrm{R}^{n}\right) / \mathscr{P}$.

Proof. Obviously, we have $X \cap \mathscr{P}=X \cap \mathscr{C}$. Therefore, it suffices to show that the constant function $F \equiv 1$ does not belong to $X$. Definition 4.2 offers two constants $C>0$ and $s_{0}>1$ such that for any $s_{0}<s,\left\|\chi_{(0,1)^{n}}\right\|_{X} \leq$ $\left\|D_{s}\right\|_{X \rightarrow X}\left\|\chi_{(0, s)^{n}}\right\|_{X} \leq C s^{-\frac{n}{2 q_{X}}}\left\|\chi_{(0, s)^{n}}\right\|_{X}$. Therefore, $s^{\frac{n}{2 q_{X}}}\left\|\chi_{(0,1)^{n}}\right\|_{X} \leq$ $C\left\|\chi_{(0, s)^{n}}\right\|_{X} \leq C\|F\|_{X}$ for any sufficiently large $s$ and, hence, $F$ does not belong to $X$.

By Theorem 4.8 with $B=\mathrm{R}$ and $a=1$ (or Theorem 5 of [42]), the HardyLittlewood maximal operator is well-defined in $X$. For any fixed $f \in X$, without loss of generality, we assume that $(\mathrm{M} f)(0)$ is well defined. Hence, for any $\varphi \in \mathscr{S}\left(\mathrm{R}^{n}\right)$, we have a semi-norm $\|\cdot\|_{N}$ in $\mathscr{S}\left(\mathrm{R}^{n}\right)$ and a constant $C>0$ so that

$$
\begin{aligned}
\left|\int_{\mathrm{R}^{n}} f(x) \varphi(x) d x\right| & \leq\|\varphi\|_{N} \sum_{k \in \mathrm{N}} \frac{k^{n}}{(1+k)^{n+2}} \frac{1}{k^{n}} \int_{k \leq|x|<k+1}|f(x)| d x \\
& \leq C\|\varphi\|_{N}(\mathrm{M} f)(0) .
\end{aligned}
$$

Thus, $f \in \mathscr{S}^{\prime}\left(\mathrm{R}^{n}\right) / \mathscr{P}$. This completes the proof.

The following result extends the Littlewood-Paley characterization to r.-i. quasi-Banach function spaces.

THEOREM 4.10. Let $X$ be a r.-i. quasi-Banach function space on $\mathrm{R}^{n}$. If the Boyd indices of $X$ satisfy $1<p_{X} \leq q_{X}<\infty$, then we have the quasi-Banach space isomorphism: $\dot{F}_{l^{2}, X}^{0}=X$.

Proof. Let $\varphi \in \mathscr{S}_{0}\left(\mathrm{R}^{n}\right)$ be a function that satisfies (2.2). Define the operator $\mathscr{G}_{\varphi}$ as $\mathscr{G}_{\varphi}(f)(x)=\left\{\left(f * \varphi_{j}\right)(x)\right\}_{j \in \mathrm{Z}}$. According to [25], ChapterV, Theorem 5.3, $\mathscr{G}_{\varphi}$ is a bounded operator from $L^{p}$ to $L_{l^{2}}^{p}$ when $1<p<\infty$. Since $1<p_{X} \leq$ $q_{X}<\infty$, there exist $1<p_{0}, q_{0}<\infty$ such that $p_{0}<p_{X} \leq q_{X}<q_{0}$. Thus, $\mathscr{G}_{\varphi}$ is of joint weak type $\left(p_{0}, q_{0}, \mathrm{C}, l^{2}\right)$. As $X \subset \mathscr{S}^{\prime}\left(\mathrm{R}^{n}\right) / \mathscr{P}, f * \varphi_{j}, j \in \mathrm{Z}$, and $\mathscr{G}_{\varphi}(f)$ are well defined for $f \in X$, we are allowed to apply Theorem 4.4 and obtain a constant $C>0$ so that $\|f\|_{\dot{F}_{l^{2}, X}^{0}}=\left\|\left(\sum_{j \in Z}\left|f * \varphi_{j}\right|^{2}\right)^{1 / 2}\right\|_{X}=$ $\left\|\mathscr{G}_{\varphi}(f)\right\|_{X_{l^{2}}} \leq C\|f\|_{X}$.

Next, we prove the other direction. Since $\varphi$ satisfies (2.2), we have a $\psi \in$ $\mathscr{S}_{0}\left(\mathrm{R}^{n}\right)$ also satisfying (2.2) such that (see [23], (4.13)) $\sum_{j \in Z} \hat{\varphi}\left(2^{-j} \xi\right) \overline{\hat{\psi}\left(2^{-j} \xi\right)}$ $=1, \xi \neq 0$. The adjoint operator of $\mathscr{G}_{\psi}, \mathscr{G}_{\psi}^{*}$, is given by $\mathscr{G}_{\psi}^{*}\left(\left\{f_{j}\right\}_{j \in \mathrm{Z}}\right)=$ $\sum_{j \in Z} \psi_{j} * f_{j}$. As $\left(L_{l^{2}}^{p}\right)^{*}=L_{l^{2}}^{p^{\prime}}, 1<p<\infty$, where $p^{\prime}$ is the conjugate of $p$, 
$\mathscr{G}_{\psi}^{*}$ is a bounded linear operator from $L_{l^{2}}^{p}$ to $L^{p}, 1<p<\infty$. Therefore, $\mathscr{G}_{\psi}^{*}$ is of joint weak type $\left(p_{0}, q_{0}, l^{2}, \mathrm{C}\right), 1<p_{0}, q_{0}<\infty$, and, hence, it is bounded from $X_{l^{2}}$ to $X$. For any $f \in \dot{F}_{l^{2}, X}^{0}$, let $f_{i}=f * \varphi_{i}, i \in \mathrm{Z}$. There exists a constant $C>0$ independent of $f$ such that $\left\|\sum_{i \in Z} \psi_{i} * \varphi_{i} * f\right\|_{X}=\left\|\mathscr{G}_{\psi}^{*}\left(\left\{f_{i}\right\}_{i \in \mathrm{Z}}\right)\right\|_{X} \leq$ $C\left\|\left\{\varphi_{i} * f\right\}_{i \in Z}\right\|_{X_{l^{2}}}=C\|f\|_{\dot{F}_{l^{2} X}^{0}}$. The Littlewood-Paley analysis yields the identity $f=\sum_{i \in \mathrm{Z}} \psi_{i} * \varphi_{i} * f$ in $\mathscr{S}^{\prime}\left(\mathrm{R}^{n}\right) / \mathscr{P}$. Therefore, our promised result follows from the condition $1<p_{X} \leq q_{X}<\infty$ and Lemma 4.9.

We investigate some important r.-i. quasi-Banach function spaces, namely, the Lorentz-Karamata spaces [16], [44], [45] and the Orlicz spaces [49].

Let $0<p, q<\infty, 0<r, u \leq \infty$ and $b$ be a slowly varying function (see [16], Definition 3.4.32). Let $l_{q, u}$ and $L_{p, r ; b}$ denote the Lorentz sequence spaces (see [55] Section 1.18.3) and the Lorentz-Karamata spaces (see [16], Definition 3.4.38), respectively. When $0<r<1$ and $1<p<\infty, L_{p, r ; b}$ are examples of r.-i. quasi-Banach function spaces with Boyd's indices located strictly in between one and infinity. Next, we show that $\left(L_{p, r ; b}, l_{q, u}\right)$ is an $a$-admissible pair if $0<a<\min (p, q, r, u)$.

Proposition 4.11. Let $0<p, q, u<\infty$ and $0<r \leq \infty$. The pair $\left(l_{q, u}, L_{p, r ; b}\right)$ is a-admissible when $0<a<\min (p, q, r, u, 1)$.

Proof. As $l_{q, u}, 1<q, u<\infty$, are reflexive r.-i. Banach sequence spaces and $\left(l_{q, u}\right)^{1 / a}=l_{q / a, u / a}$, the discussions after Definitions 1.3 and 1.5 conclude that $l_{q, u}, 0<q, u<\infty$, are regular sequence spaces. Furthermore, $l_{q, u}$, $1<q, u<\infty$, are interpolation spaces of $l^{p}, 1<p<\infty$. Proposition 1 of [50] guarantees that $l_{q, u}, 1<q, u<\infty$, possess the UMD property. In addition, the Boyd indices of $l_{q, u}$ equal to $q$ and the Boyd indices of $\left(L_{p, r ; b}\right)^{1 / a}$ equal to $p / a$. So, Theorem 4.8 shows that $\left(l_{q, u}, L_{p, r ; b}\right)$ is $a$-admissible.

Define the Triebel-Lizorkin-Lorentz spaces $\dot{F}_{p, r}^{\alpha, q, u}$ to be the LittlewoodPaley spaces $\dot{F}_{l_{q, u}, L_{p, r}}^{\alpha}$. The Triebel-Lizorkin-Lorentz spaces include some of the interpolation spaces of the Triebel-Lizorkin spaces. For instance, $\left(\dot{F}_{p_{0}}^{\alpha, q}, \dot{F}_{p_{1}}^{\alpha, q}\right)_{\theta, r}$ $=\dot{F}_{p, r}^{\alpha, q, q}, p_{0} \neq p_{1}, 1 \leq r \leq \infty$ and $\left(\dot{F}_{p_{0}}^{\alpha, q_{0}}, \dot{F}_{p_{1}}^{\alpha, q_{1}}\right)_{\theta, p}=\dot{F}_{p, p}^{\alpha, q, p}, q_{0} \neq q_{1}$, where $1 / p=(1-\theta) / p_{0}+\theta / p_{1}$ and $1 / q=(1-\theta) / q_{0}+\theta / q_{1}$ (see [56], Section 2.4.2). Moreover, the Triebel-Lizorkin-Lorentz spaces also include the Hardy-Lorentz spaces [17], weak- $H^{1}$ [19] and weak- $H^{p}$ [35] as special cases. By Theorem 4.10, $L_{p, r ; b}$ has the Littlewood-Paley characterization and, hence, $L_{p, r ; b}$ is a special case of $\dot{F}_{\mathscr{B}_{s}, \mathscr{B}_{f}}^{\alpha}$ when $1<p<\infty$. The LorentzKaramata spaces include the Lorentz-Zygmund spaces [4] and the generalized Lorentz-Zygmund spaces (see [16] p. 113).

Next, we turn to Orlicz spaces. Let $L^{\Phi}$ be the Orlicz space associated with the convex function, $\Phi$. As the Orlicz space $L^{\Phi}$ is reflexive if and only 
if $1<p_{L^{\Phi}} \leq q_{L^{\Phi}}<\infty$ (see [36], Volume II, Proposition 2.b.5 and [49] Chapter IV, Corollary 12), we have the Littlewood-Paley characterization of the reflexive Orlicz spaces $\dot{F}_{l^{2}, L^{\Phi}}^{0}=L^{\Phi}$.

We now generalize the Triebel-Lizorkin spaces by replacing $L^{p}\left(\mathrm{R}^{n}\right)$ and $l^{q}$, $1<p, q<\infty$, by the Orlicz function spaces and the Orlicz sequence spaces, respectively.

Proposition 4.12. If $l^{\Theta}$ and $L^{\Phi}$ are reflexive Orlicz sequence space and reflexive Orlicz function space, respectively, then the pair $\left(l^{\Theta}, L^{\Phi}\right)$ is admissible.

Proof. We are going to show that $\left(l^{\Theta}, L^{\Phi}\right)$ satisfies (1.2) with $a=1$. As $l^{\Theta}$ is a reflexive Orlicz sequence space (see [36], Volume I, Propositions 4.a.4 and 4.b.1), it is a regular sequence space and, moreover, $l^{\Theta}$ has the UMD property (see [20], [26], [27]). We have \|\| $\mathrm{M} f\left\|_{l^{\Theta}}\right\|_{L^{\Phi}} \leq C\|f\|_{l^{\Theta}} \|_{L^{\Phi}}$ because $1<p_{L^{\Phi}} \leq q_{L^{\Phi}}<\infty$. Finally, as the Boyd indices of $l^{\Theta}$ satisfy $1<p_{l^{\Theta}} \leq$ $q_{l^{\Theta}}<\infty$ when $l^{\Theta}$ is reflexive, Theorem 4.8 concludes that the pair $\left(l^{\Theta}, L^{\Phi}\right)$ is admissible.

From the previous result, for any $\alpha \in \mathrm{R}$ and $\Phi, \Theta$ satisfying the above conditions, the Triebel-Lizorkin-Orlicz spaces $\dot{F}_{\Theta, \Phi}^{\alpha}$ are defined as $\dot{F}_{\Theta, \Phi}^{\alpha}=\dot{F}_{l^{\Theta} L^{\Phi}}^{\alpha}$. The Triebel-Lizorkin-Orlicz spaces are extensions of the Triebel-Lizorkin spaces $\dot{F}_{p}^{\alpha, q}$ and the Orlicz spaces $L^{\Phi}$.

\section{Morrey type spaces}

The results in Section 4 may give a wrong impression to the reader that the sequence space component and the function space component of an admissible pair are restricted to the sequence spaces having the UMD property and r.-i. quasi-Banach function spaces on $\mathrm{R}^{n}$ with Lebesgue measure, respectively. In fact, the admissible pair $\left(l^{\infty}, L^{\infty}\right)$ provides a counter example on the sequence spaces and the admissible pairs $\left(l^{q}, L^{p}(\omega)\right)$ and $\left(l^{q}, L^{p(x)}\right), 1<p, q<\infty$, where $\omega$ is a Muckenhoupt $A_{p}$ weight and $L^{p(x)}$ is a variable Lebesgue space (the exponent function $p(x)$ must satisfy certain conditions, see [14]) provide counter examples on the function spaces. The reader is referred to [1] and [14] for the admissibility of $\left(l^{q}, L^{p}(\omega)\right)$ and $\left(l^{q}, L^{p(x)}\right)$, respectively. We offer another important example of non r.-i. function space that satisfies the admissibility condition, namely, the class of Morrey spaces [12], [32], [39], [52], [53].

Definition 5.1. Let $X$ be a quasi-Banach function space on $\mathrm{R}^{n}, B$ be a separable quasi-Banach lattice and $\omega(x, r): \mathrm{R}^{n} \times(0, \infty) \rightarrow(0, \infty)$ be a Lebesgue measurable function. A $B$-valued function $f \in \mathcal{M}_{0}\left(\mathrm{R}^{n}, B\right)$ belongs 
to the Morrey-type space associated with $X_{B}$ if it satisfies

$$
\|f\|_{\mathscr{M}_{\omega}^{X}(B)}=\sup _{x_{0} \in \mathrm{R}^{n}, r>0} \frac{1}{\omega\left(x_{0}, r\right)}\left\|\chi_{\mathrm{B}\left(x_{0}, r\right)} f\right\|_{X_{B}}<\infty
$$

where $\mathrm{B}\left(x_{0}, r\right)=\left\{x \in \mathrm{R}^{n}:\left|x-x_{0}\right|<r\right\}$.

For brevity, when $B=\mathrm{R}$, we write $\mathcal{M}_{\omega}^{X}(\mathrm{R})$ and $\|\cdot\|_{\mathcal{M}_{\omega}^{X}(\mathrm{R})}$ by $\mathcal{M}_{\omega}^{X}$ and $\|\cdot\|_{\mathcal{M}_{\omega}^{X}}$, respectively. Let $1<u \leq p<\infty$. If $X=L^{u}\left(\mathrm{R}^{n}\right)$ and $\omega(x, r)=r^{\frac{n}{u}-\frac{n}{p}}$, then $\mathscr{M}_{\omega}^{X}$ is the "classical" Morrey space in [12], [48]. When $\omega$ satisfies the conditions in Theorem 5.8 and $B=\mathrm{R}$, then $\mathcal{M}_{\omega}^{X}$ is the generalized Morrey space studied in [43]. When $X$ is a general r.-i. quasi-Banach function space and $\omega=1$, then $\mathscr{M}_{\omega}^{X}=X$. Let $X^{\prime}$ denote the associated space of the r.-i. Banach function space $X$ (see [5] Chapter 1, Section 2).

If $X$ is a Banach function space, it is evident that $X_{B}$ is a Banach space and, hence, $\mathscr{M}_{\omega}^{X}(B)$ is also a Banach space. The proof for the completeness of $\mathscr{M}_{\omega}^{X}(B)$ follows from the corresponding proof for the classical Morrey spaces, see [33] Section 4.4 .

THEOREM 5.2. Let $X$ be a r.-i. Banach function space on $\mathrm{R}^{n}$ with $1<p_{X} \leq$ $q_{X}<\infty$ and $B$ be a regular Banach sequence space having the UMD property. If there exists a constant $C>0$ such that for any $x \in \mathrm{R}^{n}$ and $r>0$, $\omega$ fulfills

$$
\sum_{j=0}^{\infty} 2^{-j n} r^{-n} \phi_{X^{\prime}}\left(2^{(j+1) n} r^{n}\right) \phi_{X}\left(r^{n}\right) \omega\left(x, 2^{j+1} r\right)<C \omega(x, r)
$$

where $\phi_{X}$ and $\phi_{X^{\prime}}$ are the fundamental functions of $X$ and $X^{\prime}$, respectively (see [5], Chapter 2, Definition 5.1), then there exists $C>0$ such that for any $f=\left\{f_{i}\right\}_{i \in Z} \in \mathcal{M}_{\omega}^{X}(B)$,

$$
\|\mathrm{M}(f)\|_{\mathcal{M}_{\omega}^{X}(B)} \leq C\|f\|_{\mathcal{M}_{\omega}^{X}(B)} .
$$

Proof. For any $z \in \mathrm{R}^{n}$ and $r>0$, write $f_{i}(x)=f_{i}^{0}(x)+\sum_{j=1}^{\infty} f_{i}^{j}(x)$, where $f_{i}^{0}=\chi_{\mathrm{B}(z, 2 r)} f_{i}$ and $f_{i}^{j}=\chi_{\mathrm{B}\left(z, 2^{j+1} r\right) \backslash \mathrm{B}\left(z, 2^{j} r\right)} f_{i}, j \in \mathrm{N} \backslash\{0\}$. Applying Theorem 4.8 to $f^{0}=\left\{f_{i}^{0}\right\}_{i \in Z}$, we obtain \|\| $\mathrm{M}\left(f^{0}\right)\left\|_{B}\right\|_{X} \leq C\|\| f^{0}\left\|_{B}\right\|_{X}$. We have

$$
\begin{aligned}
\frac{1}{\omega(z, r)}\left\|\chi_{\mathrm{B}(z, r)}\right\| \mathrm{M}\left(f^{0}\right)\left\|_{B}\right\|_{X} & \leq C \frac{1}{\omega(z, 2 r)}\left\|\chi_{\mathrm{B}(z, 2 r)}\right\| f\left\|_{B}\right\|_{X} \\
& \leq C \sup _{\substack{y \in \mathrm{R}^{n} \\
r>0}} \frac{1}{\omega(y, r)}\left\|\chi_{\mathrm{B}(y, r)}\right\| f\left\|_{B}\right\|_{X}
\end{aligned}
$$


because inequality (5.1) and [5], Chapter 2, Theorem 5.2 yield $\omega(z, 2 r)<$ $C \omega(z, r)$ for some $C>0$ independent of $z \in \mathrm{R}^{n}$ and $r>0$.

Since we have a constant $C>0$ so that for any $j \geq 1$ and $i \in \mathrm{Z}$,

$$
\chi_{\mathrm{B}(z, r)}(x)\left(\mathrm{M} f_{i}^{j}\right)(x) \leq C 2^{-j n} r^{-n} \chi_{\mathrm{B}(z, r)}(x) \int_{\mathrm{B}\left(z, 2^{j+1} r\right)}\left|f_{i}(y)\right| d y
$$

and $B$ is a Banach lattice, applying Theorem 3.29 of [51], we find that

$$
\begin{aligned}
\chi_{\mathrm{B}(z, r)}(x)\left\|\left\{\left(\mathrm{M} f_{i}^{j}\right)(x)\right\}_{i \in \mathrm{Z}}\right\|_{B} & \\
& \leq C 2^{-j n} r^{-n} \chi_{\mathrm{B}(z, r)}(x) \int_{\mathrm{B}\left(z, 2^{j+1} r\right)}\left\|\left\{f_{i}(y)\right\}_{i \in Z}\right\|_{B} d y .
\end{aligned}
$$

Let $\Theta(x)=\chi_{\mathrm{B}(z, r)}(x)\left\|\left\{\left(\mathrm{M} f_{i}^{j}\right)(x)\right\}_{i \in Z}\right\|_{B}$. The Hölder inequality on $X$ (see [5], Chapter 1, Theorem 2.4) yields

$$
\Theta(x) \leq C \chi_{\mathrm{B}(z, r)}(x) 2^{-j n} r^{-n}\left\|\chi_{\mathrm{B}\left(z, 2^{j+1} r\right)}\right\|_{X^{\prime}}\left\|\chi_{\mathrm{B}\left(z, 2^{j+1} r\right)}(y)\right\|\left\{f_{i}(y)\right\}_{i \in \mathrm{Z}}\left\|_{B}\right\|_{X} .
$$

By the definition of fundamental function, we assert that

$$
\Theta(x) \leq C 2^{-j n} r^{-n} \chi_{\mathrm{B}(z, r)}(x) \phi_{X^{\prime}}\left(2^{(j+1) n} r^{n}\right)\left\|\chi_{\mathrm{B}\left(z, 2^{j+1} r\right)}(y)\right\|\left\{f_{i}(y)\right\}_{i \in Z}\left\|_{B}\right\|_{X} .
$$

Applying the norm $\|\cdot\|_{X}$ on both sides of the above inequality, we deduce that

$$
\|\Theta\|_{X} \leq C 2^{-j n} r^{-n} \phi_{X^{\prime}}\left(2^{(j+1) n} r^{n}\right) \phi_{X}\left(r^{n}\right)\left\|\chi_{\mathrm{B}\left(z, 2^{j+1} r\right)}(y)\right\|\left\{f_{i}(y)\right\}_{i \in Z}\left\|_{B}\right\|_{X} .
$$

Thus,

$$
\begin{aligned}
\|\Theta\|_{X} \leq C 2^{-j n} r^{-n} \phi_{X^{\prime}}\left(2^{(j+1) n} r^{n}\right) \phi_{X}\left(r^{n}\right) \omega\left(z, 2^{j+1} r\right) \\
\sup _{\substack{y \in R^{n} \\
R>0}} \frac{1}{\omega(y, R)}\left\|\chi_{\mathrm{B}(y, R)}\right\| f\left\|_{B}\right\|_{X} .
\end{aligned}
$$

Using inequality (5.1), we obtain

$$
\begin{aligned}
\frac{1}{\omega(z, r)}\left\|\chi_{\mathrm{B}(z, r)}\right\| \mathrm{M} f\left\|_{B}\right\|_{X} & \leq \frac{1}{\omega(z, r)} \sum_{j=0}^{\infty}\left\|\chi_{\mathrm{B}(z, r)}\right\| \mathrm{M} f^{j}\left\|_{B}\right\|_{X} \\
& \leq C \sup _{\substack{y \in \mathrm{R}^{n} \\
R>0}} \frac{1}{\omega(y, R)}\left\|\chi_{\mathrm{B}(y, R)}\right\| f\left\|_{B}\right\|_{X}
\end{aligned}
$$

where the constant $C>0$ is independent of $r$ and $z$. Hence, this finishes the proof of (5.2). 
The following corollary considers a special case of condition (5.1), that special case is a well-known condition on the study of Morrey spaces.

Corollary 5.3. Let $X$ be a r.-i. Banach function space on $\mathrm{R}^{n}$ with $1<$ $p_{X} \leq q_{X}<\infty$. If $0 \leq \lambda<\frac{n}{q_{X}}$ and $\omega(x, r)$ satisfies

$$
\omega\left(x, 2^{j} r\right) \leq C 2^{j \lambda} \omega(x, r), \quad \text { for any } x \in \mathrm{R}^{n}, \quad j \in \mathrm{N} \text { and } r>0
$$

for some $C>0$, then inequality (5.1) holds.

Proof. Using Definition 4.2, we find that for any $\sigma>0$, there exists a constant $C_{\sigma}>0$ such that $\phi_{X^{\prime}}\left(2^{(j+1) n} r^{n}\right) \leq C_{\sigma} 2^{n(j+1)\left(\frac{1}{p_{X^{\prime}}}+\sigma\right)} \phi_{X^{\prime}}\left(r^{n}\right)$. By [5], Chapter 2, Theorem 5.2, we assert that $\phi_{X^{\prime}}\left(2^{(j+1) n} r^{n}\right) \phi_{X}\left(r^{n}\right) \leq$ $C_{\sigma} 2^{n(j+1)\left(\frac{1}{p_{X^{\prime}}}+\sigma\right)} r^{n}$. Thus, condition (5.3) yields

$$
\begin{aligned}
\sum_{j \in \mathrm{N}} 2^{-j n} r^{-n} \phi_{X^{\prime}}\left(2^{(j+1) n} r^{n}\right) \phi_{X}\left(r^{n}\right) & \omega\left(x, 2^{j+1} r\right) \\
\leq & C_{\sigma} \sum_{j \in \mathrm{N}} 2^{-j n\left(1-\frac{1}{p_{X^{\prime}}}-\sigma\right)+(j+1) \lambda} \omega(x, r) .
\end{aligned}
$$

Since $\lambda<\frac{n}{q_{X}}=n\left(1-\frac{1}{p_{X^{\prime}}}\right)$, we can chose a $\sigma>0$ so that $\sigma<\frac{1}{q_{X}}-\frac{\lambda}{n}$. Hence, $\omega$ satisfies (5.1).

Definition 5.4. Let $X$ be a r.-i. quasi-Banach function space on $\mathrm{R}^{n}$ with $q_{X}<\infty$. The function $\omega$ belongs to the set $\mathscr{W}_{X}$ if it satisfies (5.3) with $0 \leq$ $\lambda<\frac{n}{q_{X}}$ and there exist constants $C_{1}, C_{2}>0$ such that for any $x \in \mathrm{R}^{n}$, $\omega(x, r) \geq C_{1}, \forall r \geq 1$ and

$$
C_{2}^{-1} \leq \omega(x, t) / \omega(x, r) \leq C_{2}, \quad 0<r \leq t \leq 2 r .
$$

We now show the first main result of this section, the admissibility of $\mathscr{M}_{\omega}^{X}$.

THEOREM 5.5. Let $0<p, q \leq 1$ and $X$ be a r.-i. Banach function space on $\mathrm{R}^{n}$ with $1 \leq p_{X} \leq q_{X}<\infty$. Let $B$ be a regular Banach sequence space having the UMD property. If $\omega \in \mathscr{W}_{X^{p}}$, then for any $0<a<\min \left(p, q\left(1+\epsilon_{B}\right)\right)$, $\left(B^{q}, \mathcal{M}_{\omega}^{X^{p}}\right)$ is an a-admissible pair.

Proof. We first show that $\mathscr{M}_{\omega}^{X^{p}}$ is a regular function space. As demonstrated in the proof of Theorem 4.8, it suffices to prove that there exists a constant $C>$ 0 so that $\left\|\chi_{E}\right\|_{\mathscr{M}_{\omega}^{X}}<C$ for any $\chi_{E} \in \mathscr{M}\left(\mathrm{R}^{n}\right)$ with $|E|=1$. Let $S_{0}=\mathrm{B}(0,1)$. Using (5.3) and the definition of Boyd's indices, for any $S=\mathrm{B}\left(x, 2^{-k}\right)$ with 
$k \in \mathrm{N}$, we have a $0<\epsilon<\frac{1}{p q_{X}}-\frac{\lambda}{n}$ and constants $C_{1}, C_{2}>0$ such that

$$
\begin{aligned}
\frac{\left\|\chi_{S \cap E}\right\|_{X^{p}}}{\omega\left(x, 2^{-k}\right)} & \leq \frac{\left\|\chi_{S}\right\|_{X^{p}}}{\omega\left(x, 2^{-k}\right)} \leq C_{1} 2^{-k n\left(\frac{1}{p q_{X}}-\epsilon\right)} \frac{\left\|\chi_{S_{0}}\right\|_{X^{p}}}{\omega\left(x, 2^{-k}\right)} \\
& \leq C_{1} 2^{k \lambda-k n\left(\frac{1}{p q_{X}}-\epsilon\right)} \frac{\left\|\chi_{S_{0}}\right\|_{X^{p}}}{\omega(x, 1)}<C_{2} .
\end{aligned}
$$

As $\omega \in \mathscr{W}_{X^{p}}$, for any $S=\mathrm{B}\left(x, 2^{k}\right), k \in \mathrm{N}, \frac{1}{\omega\left(x, 2^{k}\right)}\left\|\chi_{S \cap E}\right\|_{X^{p}}<C$. Thus, condition (5.4) ensures that $\mathscr{M}_{\omega}^{X^{p}}$ is a regular function space. Since $\omega^{a} \in \mathscr{W}_{X^{p / a}}$ and $\left(\mathcal{M}_{\omega}^{X^{p}}\right)^{1 / a}=\mathscr{M}_{\omega^{a}}^{X^{p} / a}$, Theorem 5.2 shows the admissibility of $\left(B^{q}, \mathscr{M}_{\omega}^{X^{p}}\right)$.

Let $1<u \leq v<\infty$. When $0<p, q<\infty, \mathscr{B}_{s}=l^{q}, \mathscr{B}_{f}=L^{u}\left(\mathrm{R}^{n}\right)$ and $\omega(x, r)=r^{\frac{n}{p u}-\frac{n}{p v}}$, then $\dot{F}_{\mathscr{C}_{s}, \mathscr{B}_{f}}^{\alpha}$ and its Besov space counterparts are the Triebel-Lizorkin-Morrey spaces and Besov-Morrey spaces; and Morrey type Besov-Triebel spaces in [39], [52] and [53], respectively.

The following is the second main result of this section, the Littlewood-Paley characterization of $\mathscr{M}_{\omega}^{X}$. It includes the Littlewood-Paley characterization of the Morrey spaces associated with Lebesgue spaces (see Proposition 4.1 of [40]) and Theorem 4.10 (when $X$ is a Banach function space) as special cases.

THeOREM 5.6. Suppose that $X$ is a r.-i. Banach function space on $\mathrm{R}^{n}$ with $1<p_{X} \leq q_{X}<\infty$. If $\omega \in \mathscr{W}_{X}$, then we have the Banach space isomorphism $\dot{F}_{l^{2}, M_{\omega}^{X}}^{0}=\mathscr{M}_{\omega}^{X}$.

To prove the Littlewood-Paley characterization of $\mathcal{M}_{\omega}^{X}$, we have to surmount the same difficulties we encountered in Lemma 4.9 for r.-i. quasi-Banach function spaces.

Proposition 5.7. Let $X$ be a r.-i. Banach function space on $\mathrm{R}^{n}$ with $q_{X}<\infty$ and $\omega$ satisfy (5.3) with $0 \leq \lambda<\frac{n}{q_{X}}$. We have $\mathscr{M}_{\omega}^{X} \cap \mathscr{P}=\{0\}$. Moreover, if $1<p_{X} \leq q_{X}<\infty$, then $\mathscr{M}_{\omega}^{X} \subset \mathscr{S}^{\prime}\left(\mathrm{R}^{n}\right) / \mathscr{P}$.

Proof. It only needs to show that $F \equiv 1$ does not belong to $\mathscr{M}_{\omega}^{X}$. We prove by contradiction. If $F \in \mathscr{M}_{\omega}^{X}$, then for any $k \in \mathrm{N}, \chi_{\left(0,2^{k}\right)^{n}} \in \mathscr{M}_{\omega}^{X}$. Therefore, for any $\epsilon>0$, Definition 4.2 indicates that there exist $C_{1}, C_{2}>0$ so that $2^{k n\left(\frac{1}{q_{X}}-\epsilon\right)}\left\|\chi_{(0,1)^{n}}\right\|_{X} \leq C_{2}\left\|\chi_{\left(0,2^{k}\right)^{n}}\right\|_{X}, \forall k>C_{1}, k \in \mathrm{N}$. Hence, $2^{k n\left(\frac{1}{q_{X}}-\epsilon\right)} \frac{\left\|\chi_{(0,1)^{n}}\right\|_{X}}{\omega\left(0,2^{k}\right)} \leq C_{2} \frac{\left\|\chi_{\left(0,2^{k} n^{n}\right.}\right\|_{X}}{\omega\left(0,2^{k}\right)} \leq C_{2}\left\|\chi_{\left(0,2^{k}\right)^{n}}\right\|_{\mathcal{M}_{\omega}^{X}}<C_{2}\|F\|_{\mathscr{M}_{\omega}^{X}}<\infty$.

Thus, condition (5.3) produces the inequality $2^{k n\left(\frac{1}{q_{X}}-\epsilon\right)} \leq C 2^{k \lambda}$ for some $C>0$. As $\epsilon>0$ and $k \in \mathrm{N}$ are arbitrary, we have $\frac{n}{q_{X}} \leq \lambda$ which contradicts 
to the assumption $0 \leq \lambda<\frac{n}{q_{X}}$. We obtain the inclusion $\mathscr{M}_{\omega}^{X} \subset \mathscr{S}^{\prime}\left(\mathrm{R}^{n}\right) / \mathscr{P}$ by using Theorem 5.5 and the arguments in Theorem 4.10.

We now derive a boundedness result for the singular integral operators on $\mathcal{M}_{\omega}^{X}(B)$. It is a well-known result if $B=\mathrm{R}$ and $X=L^{p}, 1<p<\infty$ (see [43], Theorem 2).

Let $B_{1}$ and $B_{2}$ be Banach lattices. We call a linear operator $T: X_{B_{1}} \rightarrow$ $X_{B_{2}}$ a singular integral operator if there exists $K(x, y): B_{1} \rightarrow B_{2}$ such that $(T f)(x)=\int K(x, y) f(y) d y, x \in \mathrm{R}^{n} \backslash \operatorname{supp} f$, and $K(x, y)$ satisfies $\|K(x, y)\|_{B_{1} \rightarrow B_{2}} \leq C|x-y|^{-n}, \forall(x, y) \in \mathrm{R}^{2 n} \backslash\left\{(x, x): x \in \mathrm{R}^{n}\right\}$, for some $C>0$ independent of $x$ and $y$, where $\|\cdot\|_{B_{1} \rightarrow B_{2}}$ is the operator norm of linear operators from $B_{1}$ to $B_{2}$.

THEOREM 5.8. Let $X$ be a r.-i. Banach function space on $\mathrm{R}^{n}$ with $q_{X}<\infty$, $B_{1}$ and $B_{2}$ be separable Banach lattices. Assume that there exists a constant $C>0$ such that for any $r>0$ and $x_{0} \in \mathrm{R}^{n}, \omega$ satisfies (5.4) and

$$
\int_{r}^{\infty} \frac{\omega\left(x_{0}, t\right)}{t^{n+1}} d t \leq C \frac{\omega\left(x_{0}, r\right)}{r^{n}} .
$$

If $T$ is a singular integral operator which is bounded from $X_{B_{1}}$ to $X_{B_{2}}$, then $T$ is also bounded from $\mathcal{M}_{\omega}^{X}\left(B_{1}\right)$ to $\mathcal{M}_{\omega}^{X}\left(B_{2}\right)$.

Note that if $\omega$ satisfies (5.3) and (5.4), then $\omega$ satisfies (5.5). Theorem 5.8 generalizes the corresponding results for the Morrey spaces associated with Lebesgue spaces in [43], [47], [54]. We state a supporting lemma for Theorem 5.8. For brevity, the proof of Lemma 5.9 is omitted. The proof for the case where $X=L^{p}$ and $B=\mathrm{R}$ is given in [43], Lemma 1.

Lemma 5.9. Let $0<\delta \leq 1$. Let $X$ be a r.-i. Banach function space on $\mathrm{R}^{n}$ with $q_{X}<\infty$ and $B$ be a separable Banach lattice. If $\omega$ satisfies (5.4) and

$$
\int_{r}^{\infty} \frac{\omega\left(x_{0}, t\right)}{t^{n \delta+1}} d t \leq C \frac{\omega\left(x_{0}, r\right)}{r^{n \delta}},
$$

then there exists a constant $C>0$ such that for any $x_{0} \in \mathrm{R}^{n}$ and $r>0$, $\left\|f\left(\mathrm{M} \chi_{\mathrm{B}\left(x_{0}, r\right)}\right)^{\delta}\right\|_{X_{B}} \leq C \omega\left(x_{0}, r\right)\|f\|_{\mathcal{M}_{\omega}^{X}(B)}$.

With the above lemma, we are ready to prove Theorem 5.8 and, then, Theorem 5.6.

Proof of Theorem 5.8. Let $f \in \mathcal{M}_{\omega}^{X}\left(B_{1}\right)$. For any $x_{0} \in \mathrm{R}^{n}$ and $r>0$, write $f_{1}=f \chi_{\mathrm{B}\left(x_{0}, 2 r\right)}$ and $f_{2}=f-f_{1}$. Since $f_{1} \in X_{B_{1}}$ and $B_{2}$ is a Banach lattice, the boundedness of $T$ from $X_{B_{1}}$ to $X_{B_{2}}$ ensures that $\left\|\left(\chi_{\mathrm{B}\left(x_{0}, r\right)}\right) T f_{1}\right\|_{X_{B_{2}}} \leq$ $\|T\| \omega\left(x_{0}, 2 r\right)\|f\|_{\mathcal{M}_{\omega}^{X}\left(B_{1}\right)}$ where $\|T\|$ is the operator norm of $T$ from $X_{B_{1}}$ to $X_{B_{2}}$. 
For $f_{2}$ and $x \in \mathrm{B}\left(x_{0}, r\right)$, we use the representation $(T f)(x)=\int_{\mathrm{R}^{n}} K(x$, y) $f(y) d y$ and the definition of $\mathrm{M} \chi_{\mathrm{B}\left(x_{0}, r\right)}(y), \forall y \in \mathrm{R}^{n} \backslash \mathrm{B}\left(x_{0}, 2 r\right)$, to conclude that

$$
\left\|T f_{2}(x)\right\|_{B_{2}} \leq C \int_{\mathrm{R}^{n}} \frac{\left\|f_{2}(y)\right\|_{B_{1}}}{|x-y|^{n}} d y \leq C \frac{1}{r^{n}} \int_{\mathrm{R}^{n}}\|f(y)\|_{B_{1}} \mathrm{M} \chi_{\mathrm{B}\left(x_{0}, r\right)}(y) d y .
$$

For any $0<\delta \leq 1$, the Hölder inequality on $X$ assures that for any $x \in \mathrm{B}\left(x_{0}, r\right)$,

$$
\begin{aligned}
\left\|T f_{2}(x)\right\|_{B_{2}} & \leq C r^{-n} \int_{\mathrm{R}^{n}}\|f(y)\|_{B_{1}} \mathrm{M} \chi_{\mathrm{B}\left(x_{0}, r\right)}(y) d y \\
& \leq C r^{-n}\left\|f\left(\mathrm{M} \chi_{\mathrm{B}\left(x_{0}, r\right)}\right)^{\delta}\right\|_{X_{B_{1}}}\left\|\left(\mathrm{M} \chi_{\mathrm{B}\left(x_{0}, r\right)}\right)^{1-\delta}\right\|_{X^{\prime}} .
\end{aligned}
$$

By Lemma 2 of [43] (with $\epsilon=n-n \delta$ in that lemma), we find that $\omega$ satisfies (5.6). Lemma 5.9 leads to $\left\|\chi_{\mathrm{B}\left(x_{0}, r\right)} T f_{2}\right\|_{X_{B_{2}}} \leq C r^{-n} \omega\left(x_{0}, r\right)\|f\|_{M_{\omega}^{X}\left(B_{1}\right)}$. $\left\|\left(\mathrm{M} \chi_{\mathrm{B}\left(x_{0}, r\right)}\right)^{1-\delta}\right\|_{X^{\prime}}\left\|\chi_{\mathrm{B}\left(x_{0}, r\right)}\right\|_{X}$. Finally, we estimate $\left\|\left(\mathrm{M} \chi_{\mathrm{B}\left(x_{0}, r\right)}\right)^{1-\delta}\right\|_{X^{\prime}}$. As $\mathrm{M} \chi_{\mathrm{B}\left(x_{0}, r\right)} \leq 1$ and $\mathrm{M} \chi_{\mathrm{B}\left(x_{0}, r\right)} \leq C 2^{-k n}$ on $D_{k}$ where $D_{k}=\mathrm{B}\left(x_{0}, 2^{k+1} r\right) \backslash$ $\mathrm{B}\left(x_{0}, 2^{k} r\right), k \geq 1$, and $D_{0}=\mathrm{B}\left(x_{0}, 2 r\right)$, we assert that

$$
\begin{aligned}
\left\|\left(\mathrm{M} \chi_{\mathrm{B}\left(x_{0}, r\right)}\right)^{1-\delta}\right\|_{X^{\prime}} & \leq \sum_{k=0}^{\infty}\left\|\chi_{D_{k}}\left(\mathrm{M} \chi_{\mathrm{B}\left(x_{0}, r\right)}\right)^{1-\delta}\right\|_{X^{\prime}} \\
& \leq C \sum_{k=0}^{\infty} 2^{-k n(1-\delta)}\left\|\chi_{\mathrm{B}\left(x_{0}, 2^{k+1} r\right)}\right\|_{X^{\prime}} .
\end{aligned}
$$

By Definition 4.2, for any $\sigma>0$, there exists a constant $C_{\sigma}>0$ such that

$$
\left\|\left(\mathrm{M} \chi_{\mathrm{B}\left(x_{0}, r\right)}\right)^{1-\delta}\right\|_{X^{\prime}} \leq C_{\sigma} \sum_{k=0}^{\infty} 2^{-k n(1-\delta)+(k+1) n\left(\frac{1}{p_{X^{\prime}}}+\sigma\right)}\left\|\chi_{\mathrm{B}\left(x_{0}, r\right)}\right\|_{X^{\prime}}
$$

Let $\delta$ and $\sigma$ satisfy $\delta+\sigma<\frac{1}{q_{X}}=1-\frac{1}{p_{X^{\prime}}}$. Using [5], Chapter 2, Theorem 5.2, we have our desired result.

Proof of Theorem 5.6. Let $\varphi, \psi \in \mathscr{S}\left(\mathrm{R}^{n}\right)$ satisfy (2.2) and $\sum_{j \in Z} \hat{\varphi}\left(2^{-j} \xi\right) \overline{\hat{\psi}\left(2^{-j} \xi\right)}=1, \xi \neq 0$. The operator $\mathscr{G}_{\varphi}(f)=\left\{\varphi_{j} * f\right\}_{j \in Z}$ is a singular integral operator because the kernel $K(x, y)=\left\{\varphi_{j}(x-y)\right\}_{j \in Z}$ satisfies

$$
\begin{aligned}
\left(\sum_{j \in Z}\left|\varphi_{j}(x-y)\right|^{2}\right)^{1 / 2} & \leq\left(\sum_{j \in Z} \frac{2^{2 j n}}{\left(1+2^{j}|x-y|\right)^{2 n+2}}\right)^{1 / 2} \\
& \leq C|x-y|^{-n}, \quad x, y \in \mathrm{R}^{n}, x \neq y
\end{aligned}
$$


for some $C>0$ independent of $x$ and $y$. Therefore, Theorem 5.8 concludes that $\|f\|_{\dot{F}_{l^{2}, M_{\omega}^{X}}^{0}}=\left\|\left\{\varphi_{j} * f\right\}_{j \in Z}\right\|_{\mathscr{M}_{\omega}^{X}\left(l^{2}\right)}=\left\|\mathscr{G}_{\varphi}(f)\right\|_{\mathscr{M}_{\omega}^{X}\left(l^{2}\right)} \leq C\|f\|_{\mathcal{M}_{\omega}^{X}}, \forall f \in \mathscr{M}_{\omega}^{X}$ and, hence, the embedding $\mathscr{M}_{\omega}^{X} \hookrightarrow \dot{F}_{l^{2}, M_{\omega}^{X}}^{0}$ is valid.

Similarly, for the reserve direction $\dot{F}_{l^{2}, \mathcal{M}_{\omega}^{X}}^{0} \hookrightarrow \mathcal{M}_{\omega}^{X}$, we consider the operator $\mathscr{G}_{\psi}^{*}\left(\left\{f_{i}\right\}_{i \in \mathrm{Z}}\right)=\sum_{j \in \mathrm{Z}} \psi_{j} * f_{j}$. Theorem 4.10 ensures that $\mathscr{G}_{\psi}^{*}$ is bounded from $L_{l^{2}}^{p}$ to $L^{p}, 1<p<\infty$. Therefore, Proposition 5.7 and Theorem 5.8 yield our promised result.

ACKNOWLEDGEMENT. The author would like to thank the referee and the editor for their valuable suggestions on the context and the presentation of this paper.

\section{REFERENCES}

1. Andersen, K., and John, R., Weighted inequalities for vector-valued maximal functions and singular integrals, Studia Math. 69 (1980), 19-31.

2. Alvarez, J., Continuity of Calderón-Zygmund type operators on the predual of a Morrey space, pp. 309-319 in: Clifford Algebras in Analysis and Related Topics, Proc. Fayetteville 1993, Studies in Adv. Math., CRC, Baton Rouge, FL 1996.

3. Benedek, A., Calderón, A., and Panzone, R., Convolution operators on Banach spaces valued functions, Proc. Nat. Acad. Sci. U.S.A. 48 (1962), 356-365.

4. Bennett, C., and Rudnick, K., On Lorentz-Zygmund spaces, Dissertationes Math. 175 (1980), $1-72$.

5. Bennett, C., and Sharpley, R., Interpolation of Operators, Pure Appl. Math. 129, Academic Press, Boston, MA 1988.

6. Bergh, J., and Löfström, J., Interpolation Spaces, an Introduction, Grundlehren math. Wiss. 213, Springer, Berlin 1976.

7. Blasco, O., Ruiz, A., and Vega, L., Non-interpolation in Morrey-Campanato and Block Spaces, Ann. Scuola Norm. Sup. Pisa Cl. Sci (4) 28 (1999), 31-40.

8. Bourgain, J., Some remarks on Banach spaces in which martingale difference sequences are unconditional, Ark. Mat. 21 (1983), 163-168.

9. Bourgain, J., Extension of a result of Benedek, Calderón and Panzone, Ark. Mat. 22 (1984), 91-95.

10. Burkholder, D., A geometrical characterization of Banach spaces in which martingale difference sequences are unconditional, Ann. Probab. 9 (1981), 997-1011.

11. Burkholder, D., A geometric condition that implies the existence of certain singular integrals of Banach-space-valued-functions, pp. 270-286 in: Conference on Harmonic Analysis in Honor of Antoni Zygmund, Proc. Chicago 1982, Wadsworth, Belmont, CA 1983.

12. Campanato, S., Proprietà di una famiglia di spazi funzionali, Ann. Scuola Norm. Sup. Pisa (3) 18 (1964), 137-160.

13. Chiarenza, F., and Frasca, M., Morrey spaces and Hardy-Littlewood maximal function, Rend. Mat. Appl. (7) 7 (1987), 273-279.

14. Cruz-Uribe, D., Fiorenza, A., Martell, J., and Pérez, C., The boundedness of classical operators on variable $L^{p}$ spaces, Ann. Acad. Sci. Fenn. Math. 31 (2006), 239-264.

15. Diening, L., Hästo, P., and Roudenko, S., Function spaces of variable smoothness and integrability, J. Funct. Anal. 256 (2009), 1731-1768. 
16. Edmunds, D., and Evans, W. D., Hardy Operators, Function Spaces and Embeddings, Springer, Berlin 2004.

17. Fefferman, C., Rivière, N. M., and Sagher, Y., Interpolation between $H^{p}$ spaces: the real method, Trans. Amer. Math. Soc. 191 (1974), 75-81.

18. Fefferman, C., and Stein E. M., Some maximal inequalities, Amer. J. Math. 93 (1971), 107115.

19. Fefferman, R., and Soria F., The space weak $H^{1}$, Studia Math. 85 (1986), 1-16.

20. Fernandez, D., and Garcia, J., Interpolation of Orlicz-valued function spaces and U.M.D. property, Studia Math. 99 (1991), 23-40.

21. Frazier, M., and Jawerth, B., Decomposition of Besov spaces, Indiana Univ. Math. J. 34 (1985), 777-799.

22. Frazier, M., and Jawerth, B., The $\varphi$-transform and applications to distribution spaces, pp. $223-$ 246 in: Function Spaces and Applications, Proc. Lund 1986, Lecture Notes in Math. 1302, Springer, Berlin 1988.

23. Frazier, M., and Jawerth, B., A discrete transform and decomposition of distribution spaces, J. Funct. Anal. 93, (1990), 34-170.

24. Frazier, M., Jawerth, B., and Weiss, G., Littlewood-Paley Theory and the Study of Function Spaces, CBMS Regional Conference Series in Math. 79, Amer. Math. Soc., Providence, RI 1991.

25. García-Cuerva, J., and Rubio de Francia, J. L., Weighted Norm Inequalities and Related Topics, North-Holland Math. Studies 116, North-Holland, Amsterdam 1985.

26. Gustavsson, J. and Peetre, J., Interpolation of Orlicz spaces, Studia Math. 60 (1977), $33-59$.

27. Gutiérrez, J., On the boundedness of the Banach space-valued Hilbert transform, Dissertation, University of Texas, Austin, (1982).

28. Ho, K.-P., Annihilator, completeness and convergence of wavelet system, Nagoya Math. J. 188 (2007), 59-105.

29. Ho, K.-P., Remarks on Littlewood-Paley analysis, Canad. J. Math. 60 (2008), 1283-1395.

30. Kalton, N., Peck, N., and Roberts, J., An F-space Sampler, London Math. Soc. Lecture Note 89, Cambridge Univ. Press, Cambridge 1984.

31. Kamińska, A., Some remarks on Orlicz-Lorentz spaces, Math. Nachr. 147 (1990), 29-38.

32. Kozono, H., and Yamazaki, M., Semilinear heat equations and the Navier-Stokes equation with distributions in new function spaces as initial data, Comm. Partial Differential Equations 19 (1994), 959-1014.

33. Kufner, A., John, O., and Fučik, S., Function Spaces, Noordhoff International, Leyden 1977.

34. Kokilashvilli, V., and Krbec, M., Weighted Inequalities in Lorentz and Orlicz, Spaces, World Scientific, River Edge, NJ 1991.

35. Liu, H. P., The weak $H^{p}$ spaces on homogeneous groups, pp. 113-118 in: Harmonic Analysis, Proc. Tianjin 1988, Lecture Notes in Math. 1494, Springer, Berlin 1991.

36. Lindenstrauss, J., and Tzafriri, L., Classical Banach Spaces I and II, Ergebnisse der Math. 92 and 97, Springer, Berlin 1996.

37. Lorentz, G. G., Some new functional spaces, Ann. of Math. (2) 51 (1950), 37-55.

38. Lu, S.-Z., Four Lectures on Real H $H^{p}$ Spaces, World Scientific, Singapore 1995.

39. Mazzucato, A., Besov-Morrey spaces: Function space theory and applications to non-linear PDE, Trans. Amer. Math. Soc. 355 (2003), 1297-1364.

40. Mazzucato, A., Decomposition of Besov-Morrey spaces, pp. 279-294 in: Harmonic Analysis at Mount Holyoke, Proc. South Hadley 2001, Contemp. Math. 320, Amer. Math. Soc., Providence, RI 2003.

41. Mizuhara, T., Boundedness of some classical operators on generalized Morrey spaces, pp. 183-189 in: Harmonic Analysis, Proc. Sendai 1990, Springer, Tokyo 1991. 
42. Montgomery-Smith, S., The Hardy operator and Boyd indices, pp. 359-364 in: Interaction between Functional Analysis, Harmonic Analysis, and Probability, Proc. Columbia 1994, Lecture Notes Pure Appl. Math. 175, Dekker, New York 1996.

43. Nakai, E., Hardy-Littlewood maximal operator, singular integral operators and the Riesz potentials on generalized Morrey spaces, Math. Nachr. 166 (1994), 95-103.

44. Neves, J., Lorentz-Karamata spaces, Bessel and Riesz potientals and embeddings, Dissertationes Math. 405 (2002).

45. Neves, J., Spaces of Bessel-potential type and embeddings: the super-limiting case, Math. Nachr. 265 (2004), 68-86.

46. Okada, S., Ricker, W., and Sánchez Pérez, E., Optimal Domain and Integral Extension of Operators, Operator Theory Adv. Appl. 180, Birkhäuser, Basel 2008.

47. Peetre, J., On convolution operators leaving $L^{p, \lambda}$ spaces invariant, Ann. Mat. Pura Appl. (4) 72 (1966), 295-304.

48. Peetre, J., On the theory of $\mathscr{L}_{p, \lambda}$ spaces, J. Funct. Anal. 4 (1969), 71-87.

49. Rao, M. M., and Ren, Z. D., Theory of Orlicz Spaces, Monographs Textbooks Pure Appl. Math. 146, Dekker, New York 1991.

50. Rubio de Francia, J., Martingale and integral transforms of Banach space valued functions, pp. 195-222 in: Probability and Banach Spaces, Lecture Notes in Math. 1221, Springer, Berlin 1986.

51. Rudin, W., Functional Analysis, 2nd ed., McGraw-Hill, New York 1991.

52. Sawano, Y., and Tanaka, H., Decompositions of Besov-Morrey spaces and Triebel-LizorkinMorrey spaces, Math. Z. 257 (2007), 871-905.

53. Tang, L., and Xu, J., Some properties of Morrey type Besov-Triebel spaces, Math. Nachr. 278 (2005), 904-917.

54. Taylor, M., Analysis on Morrey Spaces and applications to Navier-Stokes and other evolution equations, Comm. Partial Differential Equations 17 (1992), 1407-1456.

55. Triebel, H., Interpolation Theory, Function Spaces, Differential Operators, North-Holland Math. Library 18, North-Holland, Amsterdam 1978.

56. Triebel, H., Theory of Function Spaces, Monographs in Math. 78, Birkhäuser, Basel 1983.

57. Zorko, C., Morrey Space, Proc. Amer. Math. Soc. 98 (1986), 586-592.

DEPARTMENT OF MATHEMATICS AND INFORMATION TECHNOLOGY

THE HONG KONG INSTITUTE OF EDUCATION

10 LO PING ROAD

TAI PO

HONG KONG

CHINA

E-mail: vkpho@ied.edu.hk 